\title{
Metric and arithmetic properties of mediant-Rosen maps
}

\author{
by \\ Cor Kraaikamp (Delft), Hitoshi Nakada (Yokohama) \\ and Thomas A. Schmidt (Corvallis, OR)
}

1. Introduction. Ergodic properties of a number-theoretic transformation can in certain circumstances be studied by way of transformations which induce it. In the classical setting of the simple continued fraction (SCF) expansion, S. Ito [8] studied maps corresponding to the mediant convergents for exactly this purpose. Motivated by this classical setting, we call any such inducing transformation a mediant map. In this paper we give mediant maps for the Rosen continued fraction maps, allowing us to extend the work of [15] and [3]. We discuss some arithmetic and metric properties of mediant convergents arising from these maps, in particular using techniques of [16] to show that the Legendre constant - determining membership in the sequence of approximations of a real number - is equal to the ergodic-theoretic Lenstra constant.

One motivation for this work comes from Diophantine approximation in terms of the Rosen fractions. Diophantine approximation by simple continued fractions has of course a rich history. In particular, for the regular continued fraction expansion we have the following classical Borel result (cf. [4]): if $x$ has SCF-expansion $x=\left[0 ; a_{1}, a_{2}, \ldots\right]$, and convergents $P_{n} / Q_{n}$, $n \geq 0$, and if the approximation coefficients $\vartheta_{n}=\vartheta_{n}(x), n \geq 0$, are defined by

$$
\vartheta_{n}=\vartheta_{n}(x)=Q_{n}^{2}\left|x-\frac{P_{n}}{Q_{n}}\right|, \quad n \geq 0,
$$

then for every $n \geq 1$ and every irrational $x$ we have

$$
\min \left(\vartheta_{n-1}, \vartheta_{n}, \vartheta_{n+1}\right)<1 / \sqrt{5},
$$

and the constant $1 / \sqrt{5}$ is sharp. Borel's result, together with the yet older Legendre result: if $p, q \in \mathbb{Z}, q>0, \operatorname{gcd}(p, q)=1$, and $|x-p / q|<1 /\left(2 q^{2}\right)$

2000 Mathematics Subject Classification: 11J70, 11K50, 37A45.

Key words and phrases: Rosen fractions, Hurwitz constants, Diophantine approximation, mediant maps, Hecke groups. 
then $p / q$ is a SCF-convergent to $x$, implies the classical Hurwitz result: for every irrational $x$ there are infinitely many rationals $p / q$, such that

$$
\left|x-\frac{p}{q}\right|<\frac{1}{\sqrt{5} q^{2}}
$$

A geometric aspect of such Diophantine approximation is expressible in terms of the Möbius action of the modular group $\operatorname{PSL}(2, \mathbb{Z})$. In the middle of the last century, this was generalized to approximation by the orbit of infinity under a reasonably large class of Fuchsian groups (see the discussion on pp. 334-336 of [11]). Some thirty years after Rosen [20] introduced his continued fractions to study elements of the Hecke triangle groups (a family of Fuchsian groups including the modular group), Lehner $[12,13]$ used these continued fractions to begin the study of the quality of approximation by the orbit of infinity under each of the Hecke groups. His goal was to use the Rosen fractions to determine the analog of the Hurwitz constant for this approximation.

These Hurwitz constants were finally determined by Haas and Series [6], using techniques of hyperbolic geometry. Let $G_{k}$ denote the Hecke group of index $k \geq 3$, generated by $z \mapsto-1 / z$ and $z \mapsto z+\lambda_{k}$ with $\lambda_{k}=2 \cos (\pi / k)$. The $G_{k}$-rationals, denoted $G_{k}(\infty)$, are the orbit of infinity under this group; a real number is called a $G_{k}$-irrational if it is not in this orbit.

Theorem 1 (Haas and Series). For $x \in \mathbb{R} \backslash G_{k}(\infty)$, let $\mu_{k}(x)=\inf \left\{h:\left|x-\frac{a}{c}\right|<\frac{h}{c^{2}}\right.$ has infinitely many solutions $\left.\frac{a}{c} \in G_{k}(\infty)\right\}$, and set $C(k)=\sup \left\{\mu_{k}(x): x \in \mathbb{R} \backslash G_{k}(\infty)\right\}$. Then

$$
C(k)= \begin{cases}1 / 2 & \text { if } k \text { is even, } \\ \frac{1}{2 \sqrt{\left(1-\lambda_{k} / 2\right)^{2}+1}} & \text { if } k \text { is odd. }\end{cases}
$$

A main goal of this paper is to give a new proof of this result using continued fraction methods and thus to complete Lehner's program. (Since $k=3$ is the index of the classical case, we will restrict our considerations to $k \geq 4$ throughout.) One could imagine that a continued fractions proof proceeds by way of analogs of Borel and Legendre results. Indeed, [9] gives a Borel-type result:

Theorem $2([9])$. For $x \in \mathbb{R} \backslash G_{k}(\infty)$ and $\left(p_{n} / q_{n}\right)_{n \geq 1}$ the corresponding Rosen convergents of $x$, one has

$$
\theta_{n}(x):=q_{n}^{2}\left|x-\frac{p_{n}}{q_{n}}\right|<C(k) \quad \text { infinitely often, }
$$

and the constant $C(k)$ is optimal. 
Furthermore, due to results of Nakada [15], a Legendre result exists for the Rosen fractions. Unfortunately, this analog of the Legendre constant is strictly less than $C(k)$, so Theorem 2 does not immediately imply Theorem 1. That is, these two results do not rule out the existence of some constant $D$, with $D<C(k)$, such that for some $G_{k}$-irrational $x$ there exist infinitely many $G_{k}$-rationals $a / c$, which are not Rosen convergents of $x$, but do satisfy

$$
\left|x-\frac{a}{c}\right|<\frac{D}{c^{2}}
$$

To address this difficulty, we introduce the mediant-Rosen maps (see Section 3). For each $k$, there is an $\ell_{k}>0$ such that (i) for any $G_{k^{-}}$irrational $x$ and any finite $G_{k}$-rational $a / c$,

$$
\left|x-\frac{a}{c}\right|<\frac{\ell_{k}}{c^{2}}
$$

implies $a / c$ is either a Rosen convergent $p_{n} / q_{n}$ for some $n \geq 0$, or a mediantRosen convergent $u_{n, l} / v_{n, l}$ of $x$; and (ii) for any $C>\ell_{k}$, there exist $x$ and $a / c$ such that

$$
\left|x-\frac{a}{c}\right|<\frac{C}{c^{2}}
$$

and $a / c$ is neither a Rosen convergent nor a mediant-Rosen convergent. We call $\ell_{k}>0$ the Legendre constant for mediant-Rosen convergents (of index $k$ ).

We turn to ergodic theory to determine the value of this Legendre constant of Diophantine approximation. In the setting of the simple continued fraction map, H. W. Lenstra, Jr., conjectured the value of the endpoint of linearity in the average value of small approximation coefficients (for almost every $x$ ); this conjecture was confirmed by Bosma et al. [2]. In Section 6 we define an analogous value, $\mathcal{L}_{k}$, the Lenstra constant for the mediant-Rosen map (of index $k$ ). (Haas [5] has recently shown that Lenstra constants of continued fraction type maps are related to universal behavior of geodesic excursions into cusps of hyperbolic surfaces.) Nakada [16] has proved that whenever a continued fraction map has a Legendre constant, then it also has a Lenstra constant, and if the map is ergodic with respect to a finite invariant measure, then these two are equal. (In the SCF case, the Legendre and Lenstra constants both equal 1/2.) We show that each mediant-Rosen map is ergodic with respect to an infinite invariant measure and hence cannot directly invoke the result of [16]. Instead, we use explicit planar extensions and the ratio ergodic theorem (see for example [1]) to prove the equality of the two constants and to determine their common value by actually evaluating the ergodic-theoretic Lenstra constant. 
TheOREM 3. Fix $k \geq 4$, and let $\ell_{k}$ and $\mathcal{L}_{k}$ be the Legendre and Lenstra constants for the mediant-Rosen maps. Furthermore, when $k$ is even then let $R$ equal 1 , otherwise let $R$ be the positive root of $x^{2}+\left(2-\lambda_{k}\right) x-1$. Then for $k>4$,

$$
\ell_{k}=\mathcal{L}_{k}=\lambda-R .
$$

Also, $\ell_{4}=\mathcal{L}_{4}=\sqrt{2} / 2$. In particular, in all cases we have

$$
\ell_{k}>C(k) \text {. }
$$

Therefore, any $G_{k}$-rational $a / c$ satisfying

$$
\left|x-\frac{a}{c}\right|<\frac{C(k)}{c^{2}}
$$

which is not a Rosen convergent must be a mediant convergent of $x$. Our next result gives a witness to the optimality of $C(k)$; we use $\Theta_{n}(x)$ to denote the coefficients of approximation for the mediant-Rosen maps, in direct analogy to the $\theta_{n}$ above (see equation (10) below for a definition).

Theorem 4. Fix $k \geq 4$. Let $C(k)$ and $R$ be as above. Then $\tau_{0}=R-\lambda$ is such that for any $C<C(k)$,

$$
\Theta_{n}\left(\tau_{0}\right)<C \quad \text { for at most finitely many } n \geq 0 .
$$

We note that Theorems 2-4 give a continued fraction proof of Theorem 1.

Outline. In the next section, we introduce the mediant algorithm. In Section 3, we give the underlying mediant maps. Section 4 is devoted to the construction of planar natural extensions for these, and to the study of their basic ergodic properties. Section 5 provides the proof of Theorem 4 . Definitions of the Legendre and Lenstra constants for the mediant maps appear in Section 6, where their equality is proven. In Section 7, we evaluate the Lenstra constants.

2. Mediants of Rosen fractions. Throughout this paper, $\lambda_{k}=$ $2 \cos (\pi / k)$ and $\mathbb{I}_{k}=[-\lambda / 2, \lambda / 2)$ for $k \geq 3$. For a fixed integer $k \geq 3$, the Rosen continued fraction map is defined by

$$
T_{k}(x)= \begin{cases}\left|\frac{1}{x}\right|-\lambda\left\lfloor\left|\frac{1}{\lambda x}\right|+\frac{1}{2}\right\rfloor, & x \neq 0, \\ 0, & x=0,\end{cases}
$$

for $x \in \mathbb{I}_{k}$; here and below, we omit the index " $k$ " whenever it is clear from context. We define

$$
\varepsilon_{n}(x)=\operatorname{sgn}\left(T^{n-1} x\right) \quad \text { and } \quad r_{n}(x)=r\left(T^{n-1} x\right)
$$




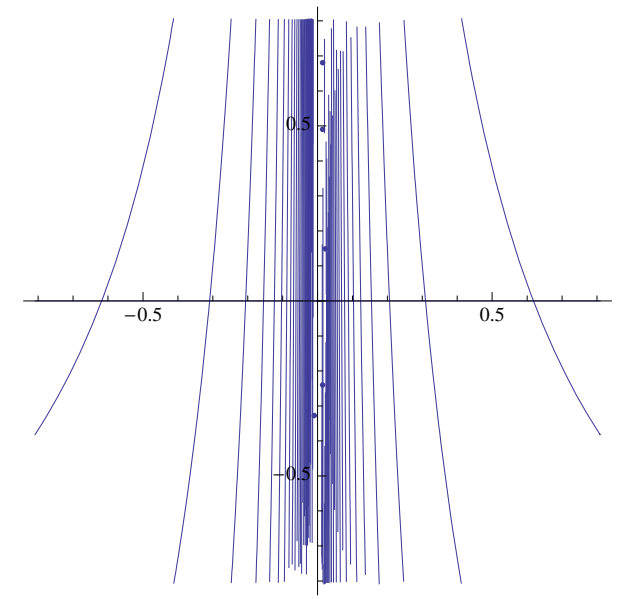

Fig. 1. Approximate graph of $T_{k}(x), k=5$

with

$$
\varepsilon(x)=\operatorname{sgn}(x) \quad \text { and } \quad r(x)=\left\lfloor\left|\frac{1}{\lambda x}\right|+\frac{1}{2}\right\rfloor .
$$

Then, as Rosen showed in [20], we have the Rosen continued fraction expansion of $x$ :

$$
x=\frac{\varepsilon_{1}(x) \mid}{\mid \lambda r_{1}(x)}+\frac{\varepsilon_{2}(x) \mid}{\mid \lambda r_{2}(x)}+\cdots+\frac{\varepsilon_{n}(x) \mid}{\mid \lambda r_{n}(x)}+\cdots,
$$

which is denoted by $x=\left[\varepsilon_{1}(x): r_{1}(x), \varepsilon_{2}(x): r_{2}(x), \ldots, \varepsilon_{n}(x): r_{n}(x), \ldots\right]$. Here the expansion terminates at a finite term if and only if $x$ is a parabolic point of $G_{k}$, thus if and only if $x$ is a $G_{k}$-rational (see [20]). As usual, we can define the convergents $p_{n} / q_{n}$ of $x \in \mathbb{I}_{k}$ by

$$
\left(\begin{array}{ll}
p_{-1} & p_{0} \\
q_{-1} & q_{0}
\end{array}\right)=\left(\begin{array}{ll}
1 & 0 \\
0 & 1
\end{array}\right)
$$

and

$$
\left(\begin{array}{cc}
p_{n-1} & p_{n} \\
q_{n-1} & q_{n}
\end{array}\right)=\left(\begin{array}{cc}
0 & \varepsilon_{1} \\
1 & \lambda r_{1}
\end{array}\right)\left(\begin{array}{cc}
0 & \varepsilon_{2} \\
1 & \lambda r_{2}
\end{array}\right) \cdots\left(\begin{array}{cc}
0 & \varepsilon_{n} \\
1 & \lambda r_{n}
\end{array}\right)
$$

for $n \geq 1$. From this definition it is easy to see that $\left|p_{n-1} q_{n}-q_{n-1} p_{n}\right|=1$, and that we have the well-known recurrence relations

$$
\begin{aligned}
& p_{-1}=1, \quad p_{0}=0, \quad p_{n}=\lambda r_{n} p_{n-1}+\varepsilon_{n} p_{n-2}, \quad n \geq 1, \\
& q_{-1}=0, \quad q_{0}=1, \quad q_{n}=\lambda r_{n} q_{n-1}+\varepsilon_{n} q_{n-2}, \quad n \geq 1 .
\end{aligned}
$$

It also follows that

$$
\left(\begin{array}{cc}
p_{n-1} & q_{n-1} \\
p_{n} & q_{n}
\end{array}\right)=\left(\begin{array}{cc}
0 & 1 \\
\varepsilon_{n} & \lambda r_{n}
\end{array}\right)\left(\begin{array}{cc}
0 & 1 \\
\varepsilon_{n-1} & \lambda r_{n-1}
\end{array}\right) \cdots\left(\begin{array}{cc}
0 & 1 \\
\varepsilon_{1} & \lambda r_{1}
\end{array}\right)
$$


giving

$$
\frac{p_{n}}{q_{n}}=\frac{\varepsilon_{1} \mid}{\mid \lambda r_{1}}+\frac{\varepsilon_{2} \mid}{\mid \lambda r_{2}}+\cdots+\frac{\varepsilon_{n} \mid}{\mid \lambda r_{n}}
$$

and

$$
\frac{q_{n-1}}{q_{n}}=\frac{1}{\mid \lambda r_{n}}+\frac{\varepsilon_{n} \mid}{\mid \lambda r_{n-1}}+\cdots+\frac{\varepsilon_{2} \mid}{\mid \lambda r_{1}} .
$$

Since Hecke groups are discontinuous groups, the (parabolic) value $p_{n} / q_{n}$ uniquely determines $q_{n}$ up to sign; we can and do assume $q_{n}$ to be positive.

From the definition of $p_{n} / q_{n}$, we have

$$
\frac{p_{n+1}}{q_{n+1}}=\frac{r_{n+1} \lambda p_{n}+\varepsilon_{n+1} p_{n-1}}{r_{n+1} \lambda q_{n}+\varepsilon_{n+1} q_{n-1}} .
$$

If $r_{n+1}>1$, then - following [8] in the SCF-case - we can interpolate between $p_{n} / q_{n}$ and $p_{n+1} / q_{n+1}$ by

$$
\frac{u_{n, l}}{v_{n, l}}=\frac{l \lambda p_{n}+\varepsilon_{n+1} p_{n-1}}{l \lambda q_{n}+\varepsilon_{n+1} q_{n-1}}, \quad 1 \leq l<r_{n+1},
$$

and call $u_{n, l} / v_{n, l}$ the $l$ th mediant convergent of $x$ (of level $n$ ); note that such a mediant does not exist in case $r_{n+1}=1$. In the next section, we define a map which induces mediant convergents of Rosen continued fractions.

\section{Mediant maps and convergents}

3.1. Full mediant map. For each fixed $k$, put $\mathbb{J}=\mathbb{J}_{k}=[-\lambda / 2,2 / \lambda)$.

For ease of notation, we let $\left(\begin{array}{ll}a & b \\ c & d\end{array}\right)(x)$ denote $\frac{a x+b}{c x+d}$. This allows us to show a "factorization" of the map $T_{k}$, leading to our definition of the mediant-Rosen maps.

The following is trivially verified.

LEMMA 1. The Rosen map $T_{k}$ can be expressed in the following manner.

$$
T_{k}(x)= \begin{cases}\left(\begin{array}{cc}
\lambda & 1 \\
-1 & 0
\end{array}\right)(x), & x \in\left[-\frac{\lambda}{2},-\frac{2}{3 \lambda}\right), \\
\left(\begin{array}{cc}
t \lambda & 1 \\
-1 & 0
\end{array}\right)(x), & x \in\left[-\frac{2}{(2 t-1) \lambda},-\frac{2}{(2 t+1) \lambda}\right), t \in \mathbb{N}_{\geq 2}, \\
\left(\begin{array}{cc}
-t \lambda & 1 \\
1 & 0
\end{array}\right)(x), & x \in\left(\frac{2}{(2 t+1) \lambda}, \frac{2}{(2 t-1) \lambda}\right], t \in \mathbb{N}_{\geq 2}, \\
\left(\begin{array}{cc}
-\lambda & 1 \\
1 & 0
\end{array}\right)(x), & x \in\left(\frac{2}{3 \lambda}, \frac{\lambda}{2}\right) .\end{cases}
$$


Definition 1. We define the matrices

$$
U_{-}=\left(\begin{array}{cc}
0 & -1 \\
1 & \lambda
\end{array}\right), \quad U_{+}=\left(\begin{array}{cc}
0 & 1 \\
1 & \lambda
\end{array}\right), \quad V_{-}=\left(\begin{array}{cc}
-1 & 0 \\
\lambda & 1
\end{array}\right), \quad V_{+}=\left(\begin{array}{cc}
1 & 0 \\
\lambda & 1
\end{array}\right) .
$$

The inverses of these are of course:

$$
U_{-}^{-1}=\left(\begin{array}{cc}
\lambda & 1 \\
-1 & 0
\end{array}\right), \quad U_{+}^{-1}=\left(\begin{array}{cc}
-\lambda & 1 \\
1 & 0
\end{array}\right), \quad V_{-}^{-1}=\left(\begin{array}{cc}
-1 & 0 \\
\lambda & 1
\end{array}\right), \quad V_{+}^{-1}=\left(\begin{array}{cc}
1 & 0 \\
-\lambda & 1
\end{array}\right) .
$$

The next lemma is verified by direct computation.

Lemma 2. The following equalities hold:

$$
\left(\begin{array}{cc}
t \lambda & 1 \\
-1 & 0
\end{array}\right)=U_{+}^{-1} \cdot V_{+}^{-(t-2)} \cdot V_{-}^{-1} \quad \text { and } \quad\left(\begin{array}{cc}
-t \lambda & 1 \\
1 & 0
\end{array}\right)=U_{+}^{-1} \cdot V_{+}^{-(t-1)} .
$$

Definition 2. The mediant-Rosen map is

$$
S_{k}(x)= \begin{cases}U_{-}^{-1}(x), & x \in\left[-\frac{\lambda}{2},-\frac{2}{3 \lambda}\right), \\ V_{-}^{-1}(x), & x \in\left[-\frac{2}{3 \lambda}, 0\right), \\ V_{+}^{-1}(x), & x \in\left(0, \frac{2}{3 \lambda}\right], \\ U_{+}^{-1}(x), & x \in\left(\frac{2}{3 \lambda}, \frac{2}{\lambda}\right) .\end{cases}
$$

In the case of $k=3, T_{3}$ is the classical nearest integer continued fraction map, and $S_{3}$ is the mediant map as defined by R. Natsui in [18]. In what follows, we always assume that $k \geq 4$. Here also, we suppress the index $k$ when discussing these maps.

Direct calculation also shows the following.

LEMMA 3. Viewed as a linear fractional transformation, the matrix $V_{-}^{-1}$ is a decreasing bijective map from $\left(-\frac{2}{(2 l-1) \lambda},-\frac{2}{(2 l+1) \lambda}\right)$ to $\left(\frac{2}{(2 l-1) \lambda}, \frac{2}{(2 l-3) \lambda}\right)$. Similarly, the linear fractional transformation $V_{+}^{-1}$ is an increasing bijective map from $\left(\frac{2}{(2 l+1) \lambda}, \frac{2}{(2 l-1) \lambda}\right)$ to $\left(\frac{2}{(2 l-1) \lambda}, \frac{2}{(2 l-3) \lambda}\right)$.

Finally, $T(x)$ is induced by $S(x)$ :

Lemma 4. For each $x \in \mathbb{I}_{k}$, let $\ell(x)$ be defined as follows.

$$
\ell(x):=\min \left\{l \geq 0: S^{l}(x) \in\left[-\frac{\lambda}{2},-\frac{2}{3 \lambda}\right) \cup\left(\frac{2}{3 \lambda}, \frac{2}{\lambda}\right)\right\} .
$$

Then for each $x \in \mathbb{I}_{k}$,

$$
S^{\ell(x)+1}(x)=T(x) .
$$

Proof. This follows from our definitions and from the lemmas of this section. 


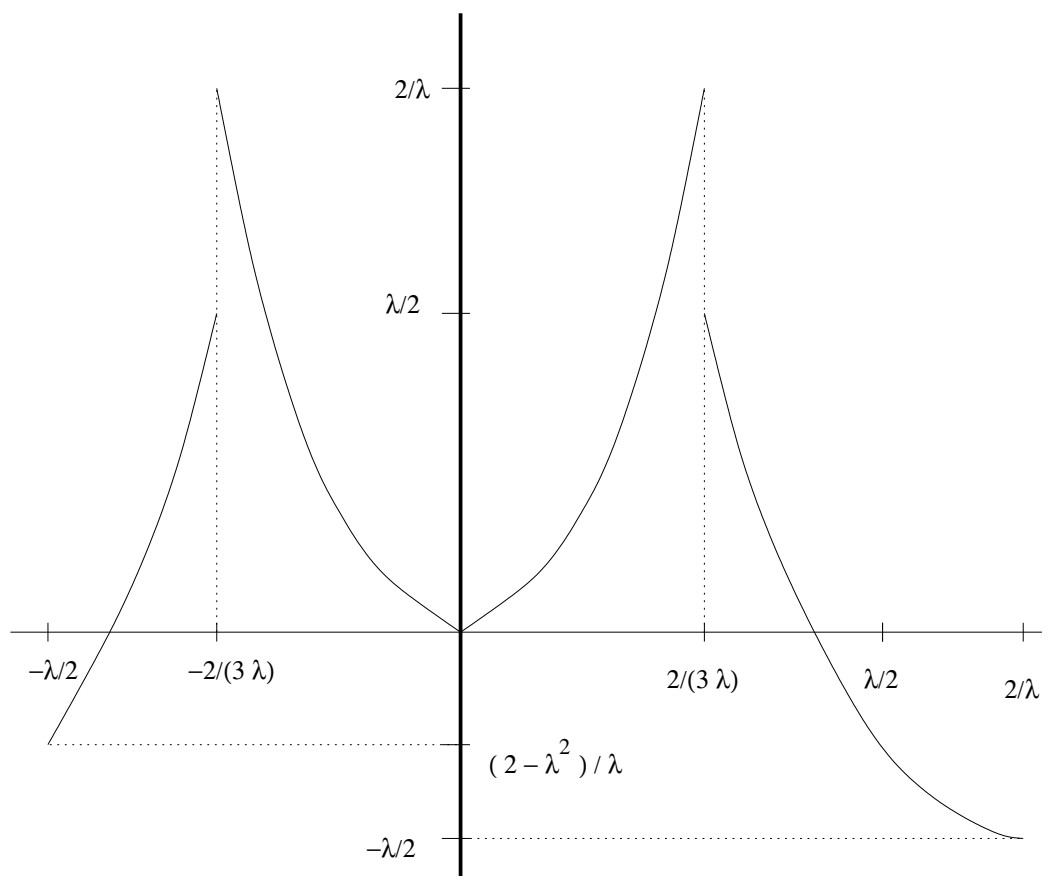

Fig. 2. Schematic representation of the graph of $S_{k}(x)$

3.2. Mediant convergents. For $x \in \mathbb{I}_{k}$ and $i \in \mathbb{N}$, we let

$$
M_{i}= \begin{cases}U_{-} & \text {if } S^{i-1}(x) \in\left[-\frac{\lambda}{2},-\frac{2}{3 \lambda}\right), \\ U_{+} & \text {if } S^{i-1}(x) \in\left(\frac{2}{3 \lambda}, \frac{2}{\lambda}\right), \\ V_{-} & \text {if } S^{i-1}(x) \in\left[-\frac{2}{3 \lambda}, 0\right), \\ V_{+} & \text {if } S^{i-1}(x) \in\left(0, \frac{2}{3 \lambda}\right], \\ \text { Id } & \text { if } S^{i-1}(x)=0,\end{cases}
$$

for $i \geq 1$, where as usual Id denotes the identity.

Then we have a sequence of matrices from $x$ which is denoted by

$$
x \sim M_{1} \cdots M_{j} \cdots
$$

Of course, the mediant map acts as a shift on each such sequence.

Definition 3. Fix $x \in \mathbb{I}_{k}$ and consider the above sequence of $M_{i}$. Let $k_{1}, k_{2}, \ldots$ be the increasing sequence of indices for which $M_{k_{i}} \in\left\{U_{-}, U_{+}\right\}$.

The following lemma records the fact that the sequence of Rosen convergents $p_{n} / q_{n}$ of $x \in \mathbb{I}_{k}$ is a subsequence of the sequence $u_{n, l} / v_{n, l}, n \geq 1$, of mediant convergents of $x$. 
Lemma 5. For each $x \in \mathbb{I}_{k}$, consider the corresponding sequence of equation (4). Then, for each $k_{m}$ as above,

$$
M_{1} \cdots M_{k_{m}}=\left(\begin{array}{cc}
p_{m-1} & p_{m} \\
q_{m-1} & q_{m}
\end{array}\right) .
$$

Furthermore, $k_{m+1}=k_{m}+r_{m+1}$ where $\varepsilon_{m}: r_{m}$ is the mth coefficient of the Rosen continued fraction expansion of $x$.

Definition 4. Let $x, m$ and $k_{m}$ be as above. For each integer $l$ with $0<l<r_{m+1}$, we define

$$
\left\{\begin{array}{l}
u_{m, l}=l \lambda p_{m}+\varepsilon_{m+1} p_{m-1} \\
v_{m, l}=l \lambda q_{m}+\varepsilon_{m+1} q_{m-1}
\end{array}\right.
$$

and call $u_{m, l} / v_{m, l}$ an $l$ th mediant convergent of $x$.

We have the following result.

Proposition 1. With notation as above, we have

$$
M_{1} \cdots M_{k_{m}} \cdots M_{k_{m}+l}=\left(\begin{array}{cc}
u_{m, l} & p_{m} \\
v_{m, l} & q_{m}
\end{array}\right)
$$

for $1 \leq l<r_{m+1}$.

By this proposition, we see that the sequence $\left(M_{1} \cdots M_{i}(\infty): i \geq 1\right)$ is

$$
\begin{aligned}
\frac{u_{0,1}}{v_{0,1}}, \frac{u_{0,2}}{v_{0,2}}, \ldots, \frac{u_{0, r_{1}-1}}{v_{0, r_{1}-1}}, \frac{p_{0}}{q_{0}}, \frac{u_{1,1}}{v_{1,1}}, \frac{u_{1,2}}{v_{1,2}}, \ldots, \frac{u_{1, r_{2}-1}}{v_{1, r_{2}-1}}, \frac{p_{1}}{q_{1}}, \ldots \\
\quad \ldots, \frac{u_{n, 1}}{v_{n, 1}}, \frac{u_{n, 2}}{v_{n, 2}}, \ldots, \frac{u_{n, r_{n+1}-1}}{v_{n, r_{n+1}-1}}, \frac{p_{n}}{q_{n}}, \ldots
\end{aligned}
$$

It is easy to see that

$$
x=\left(\begin{array}{ll}
u_{m, l} & p_{m} \\
v_{m, l} & q_{m}
\end{array}\right)\left(S^{n}(x)\right)
$$

for $n=k_{m}+l$. We put $x_{n}=S^{n}(x)$. It follows that

$$
\left|x-\frac{u_{m, l}}{v_{m, l}}\right|=\left|\frac{u_{m, l} x_{n}+p_{m}}{v_{m, l} x_{n}+q_{m}}-\frac{u_{m, l}}{v_{m, l}}\right|=\frac{1}{v_{m, l}^{2}\left(x_{n}-\left(-q_{m} / v_{m, l}\right)\right)},
$$

where $-q_{m} / v_{m, l}=\left(M_{1} \cdots M_{n}\right)^{-1}(\infty)$. We recall that $\left(M_{1} \cdots M_{n}\right)^{-1}$ is the linear fractional transformation which defines $S^{n}$. It also follows that

$$
\left|x-\frac{p_{m-1}}{q_{m-1}}\right|=\frac{1}{q_{m-1}^{2}\left(x_{k_{m}}-\left(-q_{m} / q_{m-1}\right)\right)}
$$

where $-q_{m} / q_{m-1}=\left(M_{1} \cdots M_{k_{m}}\right)^{-1}(\infty)$. Consequently, the distribution of

$$
\left(\left(M_{1} \cdots M_{n}\right)^{-1}(x)-\left(M_{1} \cdots M_{n}\right)^{-1}(\infty): n \geq 1\right)
$$

determines the distribution of the error (after normalization by the square of the denominator) of the principal and the mediant convergents. 
If $k=3$, since $\lambda_{3}=1$, it is easy to see that

$$
\left(\begin{array}{l}
u_{m, 1} \\
v_{m, 1}
\end{array}\right)=\left(\begin{array}{l}
u_{m-1, r_{m}-1} \\
v_{m-1, r_{m}-1}
\end{array}\right)
$$

when $\varepsilon_{m+1}=-1$. This equality never holds for $k \geq 4$. Indeed, since $\lambda_{k}>1$ for $k \geq 4$, we have, due to (3),

$$
v_{m, 1}=\lambda q_{m}-q_{m-1}>q_{m}-\lambda q_{m-1}=v_{m-1, r_{m}-1} .
$$

This implies that all values in

$$
\left\{\frac{u_{m, l}}{v_{m, l}}, \frac{p_{m}}{q_{m}}\right\}_{m \geq 0}
$$

are different from each other.

4. Natural extensions. Since the work of [17], planar natural extensions of continued fractions maps have provided a significant tool in the study of ergodic properties of number-theoretic transformations. In this section we construct planar natural extensions of the mediant-Rosen maps.

In [19], Rokhlin introduced and studied the concept of natural extension of a dynamical system. A natural extension of $(\mathbb{J}, \mathcal{B}, \mu, T)$ is an invertible dynamical system $\left(\Omega, \mathcal{B}_{\Omega}, \rho, \hat{T}\right)$, which contains $(\mathbb{J}, \mathcal{B}, \mu, T)$ as a factor, such that the Borel $\sigma$-algebra $\mathcal{B}_{\Omega}$ of $\Omega$ is the smallest $\hat{T}$-invariant $\sigma$-algebra that contains $\pi^{-1}(\mathcal{B})$, where $\pi$ is the factor map. A natural extension is unique up to isomorphism. With notation defined below, we have the following result.

TheOREM 5. For $k \geq 4$, the dynamical system $\left(\Omega^{*}, \overline{\mathcal{B}}, \nu, \hat{S}\right)$ is the natural extension of the dynamical system $\left(\mathbb{J}_{k}, \mathcal{B}, \mu_{k}, S\right)$.

There are various ways to verify that a planar system is the natural extension of a given interval map; one way is to follow the proof from [14], where the second author shows that the two-dimensional regions he finds are indeed the natural extensions of his $\alpha$-expansions. His proof closely follows [19]. Here, however, we turn to F. Schweiger's [22] formalization of the ideas of [17] to verify that we have found the natural extension.

In our construction, we rely on [3]. However, we proceed slightly differently. There the explicit natural extension map is

$$
\mathcal{T}(x, y)=\left(T(x), \frac{1}{\lambda\left\lfloor\left|\frac{1}{\lambda x}\right|+\frac{1}{2}\right\rfloor+\operatorname{sgn}(x) y}\right),
$$

which is locally of the form given by

$$
M=\left(\begin{array}{ll}
a & b \\
c & d
\end{array}\right) \quad \text { sending }(x, y) \text { to }\left(\frac{a x+b}{c x+d}, \frac{d y-c}{-b y+a}\right)
$$


an elementary calculation thus shows that this map has invariant measure $d x d y /(1+x y)^{2}$, up to normalizing constants. Here, we use the more natural action directly related to hyperbolic geometry:

$$
(x, y) \mapsto\left(\frac{a x+b}{c x+d}, \frac{a y+b}{c y+d}\right) .
$$

These maps are conjugate, via $(x, y) \mapsto(x,-1 / y)$, thus there is no loss in proceeding in our manner. The invariant measure for our map is well known to be $d x d y /(x-y)^{2}$. The domain $\Omega$ of $\mathcal{T}$ is defined (depending on parity) in Theorem 3.1 and Theorem 3.2 of [3]; up to measure zero, $\mathcal{T}(\Omega)=\Omega$. We let

$$
\Omega_{0}=\{(x, y):(x,-1 / y) \in \Omega\}
$$

and hence $\Omega_{0}$ is an isomorphic copy of the region of the natural extension of the Rosen map $T$.

The parity of the index $k$ is significant, as in particular displayed by the orbit of $\pm \lambda / 2$ under $S$; we thus discuss the even index case and the odd index case separately.

4.1. Planar system in the even index case: $k=2 l$. We recall some notations from [3]. Let

$$
\phi_{0}=-\frac{\lambda}{2}, \quad \phi_{j}=T^{j}\left(-\frac{\lambda}{2}\right), \quad 0 \leq j \leq l-1, \quad \phi_{l-1}=0,
$$

and

$$
L_{1}=\frac{1}{\lambda+1}, \quad L_{j}=\frac{1}{\lambda-L_{j-1}}, \quad 2 \leq j \leq l-1, \quad 1=\frac{1}{\lambda-L_{l-1}} .
$$

It is in terms of these various $\phi_{i}$ and $L_{i}$ that [3] defines the domain $\Omega$.

Now, for $1 \leq j \leq l-1$, let

$$
\begin{aligned}
J_{j} & =\left[\phi_{j-1}, \phi_{j}\right), & \bar{K}_{j} & =\left[-\infty,-1 / L_{j}\right], \\
J_{l} & =[0, \lambda / 2), & \bar{K}_{l} & =[-\infty, 0], \\
J_{l+1} & =[\lambda / 2,2 / \lambda), & \bar{K}_{l+1} & =[-1,0],
\end{aligned}
$$

and let $K_{j}^{\prime}=\bar{K}_{j}$ for $1 \leq j \leq l-1$ as well as $K_{l}^{\prime}=[-\infty,-1]$. Solving, we find

$$
\Omega_{0}=\bigcup_{j=1}^{l}\left(J_{j} \times K_{j}^{\prime}\right) .
$$

We define the region (of the natural extension for the mediant map)

$$
\Omega^{*}=\bigcup_{j=1}^{l+1}\left(J_{j} \times \bar{K}_{j}\right)
$$


(see Figure 3). The map $\hat{S}: \Omega^{*} \rightarrow \Omega^{*}$ is given by

$$
\hat{S}(x, y)=\left(M_{1}^{-1}(x), M_{1}^{-1}(y)\right),
$$

where $M_{1}=M_{1}(x)$ as in equation (4). In particular, the projection onto the first coordinate is indeed $M_{1}^{-1}(x)=S(x)$.
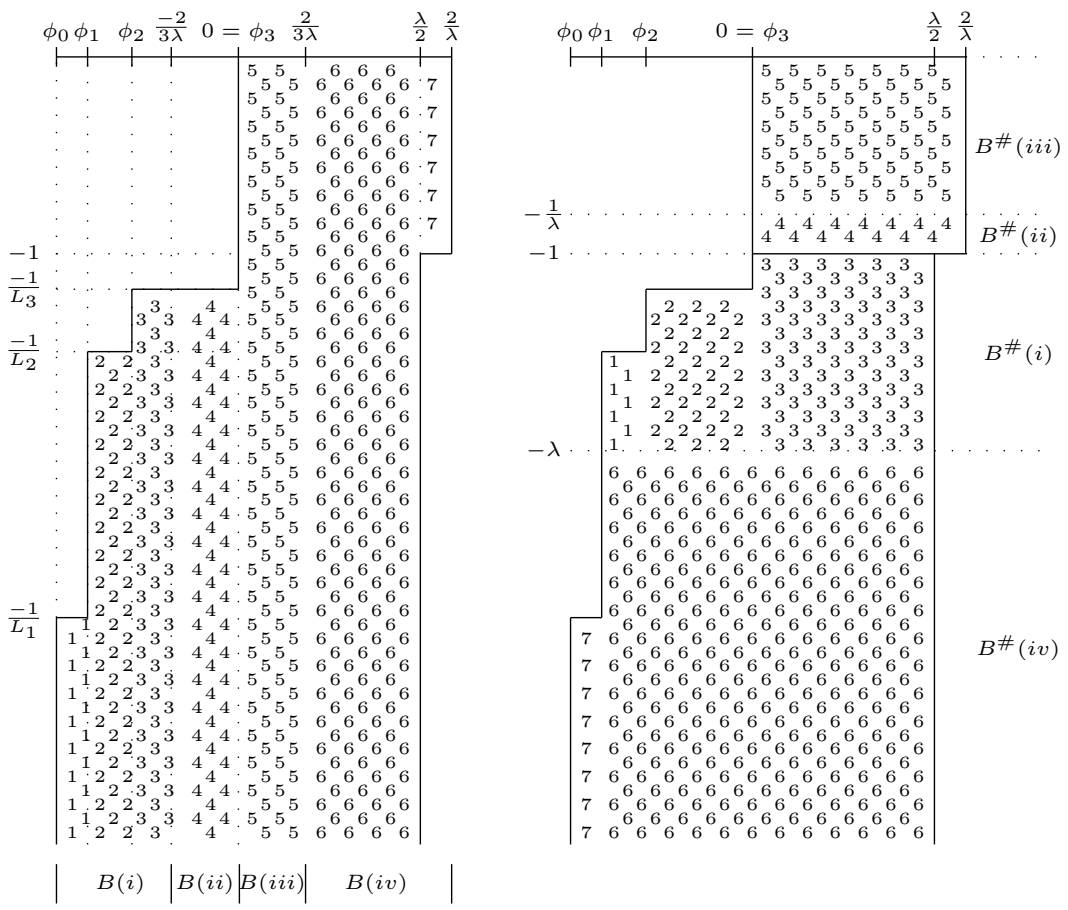

Fig. 3. The region $\Omega^{*}$ and its image under $\hat{S}$, here $k=8$. Note that $\Omega_{0}$ lies below $y=-1$.

Proposition 2. The map $\hat{S}$ is surjective from $\Omega^{*}$ onto itself and is injective off the boundaries of $J_{j} \times \bar{K}_{j}, 1 \leq j \leq l+1$. Moreover, $d x d y /|x-y|^{2}$ is an invariant measure for $\hat{S}$.

Proof. We must simply check that, up to measure zero niceties, the map $\hat{S}(x, y)$ does indeed act bijectively on $\Omega^{*}$. This is a matter of elementary calculations, which we now outline.

- $x \in[-\lambda / 2,-2 /(3 \lambda))$ : On this interval, $S(x)=-1 / x-\lambda$. Recall that the $\phi_{i}$ are in fact the orbit of $\phi_{0}=-\lambda / 2$ under iteration of this map; in particular, $\phi_{l-1}=0$. Then $\hat{S}$ sends $\bigcup_{i=1}^{l-1}\left[\phi_{i-1}, \phi_{i}\right) \times\left[-\infty,-1 / L_{i}\right]$ to $\bigcup_{i=1}^{l-1}\left[\phi_{i}, \phi_{i+1}\right) \times\left[-\lambda_{q},-1 / L_{i+1}\right]$. Also $\left[\phi_{l-1},-2 /(3 \lambda)\right) \times\left[-\infty,-1 / L_{l-1}\right]$ is now sent to $[0, \lambda / 2) \times[-\lambda,-1]$.

- $x \in[-2 /(3 \lambda), 0)$ : On this interval, $\hat{S}(x, y)=(-x /(\lambda x+1),-y /(\lambda y+1))$ for $(x, y) \in \Omega^{*}$. Since $L_{l-1}=\lambda-1$, we easily find that $\hat{S}$ sends $[-2 /(3 \lambda), 0) \times\left[-\infty,-1 / L_{l-1}\right]$ to $[0,2 / \lambda) \times[-1,-1 / \lambda]$. 
- $x \in[0,2 /(3 \lambda))$ : On this interval, $\hat{S}(x, y)=(x /(1-\lambda x), y /(1-\lambda y))$ for $(x, y) \in \Omega^{*}$. One immediately finds that $[0,2 /(3 \lambda)) \times[-\infty, 0]$ is sent to $[0,2 / \lambda) \times[-1 / \lambda, 0]$.

- $x \in[2 /(3 \lambda), \lambda / 2)$ : On this interval, $\hat{S}(x, y)=(1 / x-\lambda, 1 / y-\lambda)$ for $(x, y) \in \Omega^{*}$. One finds that $[2 /(3 \lambda), \lambda / 2) \times[-\infty, 0)$ is sent to $\left[\phi_{1}, \lambda / 2\right) \times$ $[-\infty,-\lambda]$.

- $x \in[\lambda / 2,2 / \lambda)$ : On this interval, also $\hat{S}(x, y)=(1 / x-\lambda, 1 / y-\lambda)$ for $(x, y) \in \Omega^{*}$. Hence $[2 /(3 \lambda), \lambda / 2) \times[-1,0]$ is sent to $\left[-\lambda / 2, \phi_{1}\right) \times$ $[-\infty,-\lambda-1]$.

Consequently, we see that $\hat{S}$ is bijective except for failing to be injective on the (measure zero) boundaries. The invariance of the measure holds since $\hat{S}$ is locally of the form $(x, y) \mapsto(A x, A y)$ with $A$ a fractional linear transformation.

REMARK. Note that

$$
\iint_{\Omega^{*}} \frac{d x d y}{|x-y|^{2}}=\infty
$$

4.2. Planar system in the odd index case, $k=2 l+3$. We recycle notation, now using $\phi_{j}$ and $L_{j}$ as follows (all necessary calculations are in [3]):

$$
\phi_{0}=-\frac{\lambda}{2}, \quad \phi_{j}=T^{j}\left(-\frac{\lambda}{2}\right), 0 \leq j \leq 2 l+1 .
$$

We recall that

$$
\left\{\begin{array}{l}
-\frac{\lambda}{2} \leq \phi_{j}<-\frac{2}{3 \lambda} \quad \text { for } j \in\{0,1, \ldots, l-1\} \cup\{l+1, \ldots, 2 l\}, \\
-\frac{2}{3 \lambda}<\phi_{l}<-\frac{2}{5 \lambda} \\
\phi_{2 l+1}=0 .
\end{array}\right.
$$

Also we put, with $R$ the positive root of $R^{2}+(2-\lambda) R-1=0$,

$$
\begin{aligned}
& L_{2 l}=\lambda-1 / R, \quad L_{2 l+1}=\lambda-R, \\
& L_{1}=\frac{1}{2 \lambda-L_{2 l}}, \quad L_{2}=\frac{1}{2 \lambda-L_{2 l+1}}, \quad L_{j}=\frac{1}{\lambda-L_{j-2}}, \quad 2<j \leq 2 l+2,
\end{aligned}
$$

which are well-defined (see Subsection 3.2 of [3]). Then we define

$$
\Omega^{*}=\bigcup_{j=1}^{2 l+4} J_{j} \times \bar{K}_{j}
$$

where

$$
\begin{aligned}
J_{2 j} & =\left[\phi_{l+j}, \phi_{j}\right), \quad 1 \leq j \leq l, \\
J_{2 j-1} & =\left[\phi_{j-1}, \phi_{j+l}\right), \quad 1 \leq j \leq l+1, \quad J_{2 l+4}=[1,2 / \lambda), \\
J_{2 l+2} & =[0, \lambda / 2), \quad J_{2 l+3}=[\lambda / 2,1), \quad
\end{aligned}
$$




$$
\begin{aligned}
& 1=-\phi_{l} /\left(\lambda \phi_{l}+1\right), \text { and } \\
& \bar{K}_{j}=\left[-\infty,-1 / L_{j}\right], \quad 1 \leq j \leq 2 l+1, \quad \bar{K}_{2 l+2}=[-\infty, 0], \\
& \bar{K}_{2 l+3}=\left[-\frac{1}{\lambda-L_{2 l+1}}, 0\right]=[-1 / R, 0], \quad \bar{K}_{2 l+4}=\left[-\frac{1}{\lambda-L_{2 l} l}, 0\right]=[-R, 0]
\end{aligned}
$$

(see Figure 4).
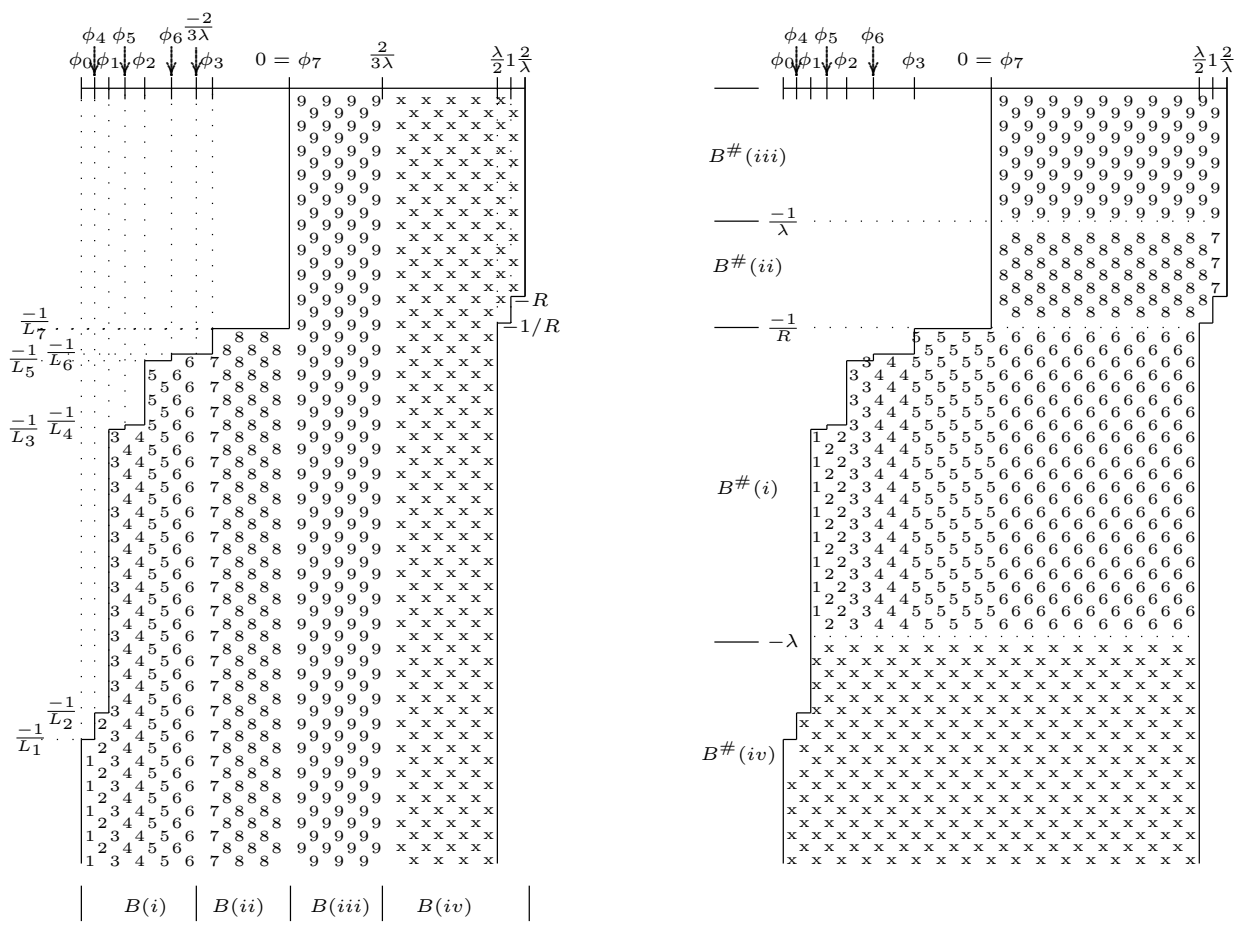

Fig. 4. The region $\Omega^{*}$ and its image under $\hat{S}$, here $k=9$. Here, $\Omega_{0}$ lies below $y=-1 / R$.

Proposition 3. The map $\hat{S}(x, y)=\left(M_{1}^{-1}(x), M_{1}^{-1}(y)\right)$ of $\Omega^{*}$ is bijective off the boundaries of $J_{j} \times \bar{K}_{j}, 1 \leq j \leq 2 l+2$. Moreover, $d x d y /|x-y|^{2}$ is an invariant measure for $\hat{S}$.

Proof. The invariance of the measure has already been remarked upon. The first part of the assertion follows as in Proposition 2:

- $x \in[-\lambda / 2,-2 /(3 \lambda))$ : Here, $\hat{S}(x, y)=(-1 / x-\lambda,-1 / y-\lambda)$. Thus, the corresponding image of $\Omega^{*}$ is fibred below $\left[\phi_{1}, \lambda / 2\right)$, with $y$ values in $\left[-\lambda,-1 / L_{j}\right]$ for $S(x)$ negative, with the appropriate value of $L_{j}$, and $y \in[-\lambda,-1 / R]$ for $S(x)$ non-negative.

- $x \in[-2 /(3 \lambda), 0)$ : Here $\hat{S}(x, y)=(-x /(\lambda x+1),-y /(\lambda y+1))$ for $(x, y)$ $\in \Omega^{*}$. Thus $[-2 /(3 \lambda), 0) \times\left[-\infty,-1 / L_{2 l+1}\right]$ is sent to $[0,2 / \lambda) \times$ $\left[-1 /\left(\lambda-L_{2 l+1}\right),-1 / \lambda\right]$. 
- $x \in[0,2 /(3 \lambda))$ : For these values of $x, \hat{S}(x, y)=(x /(1-\lambda x), y /(1-\lambda y))$ for $(x, y) \in \Omega^{*}$. Thus $[0,2 /(3 \lambda)) \times[-\infty, 0]$ is sent to $[0,2 / \lambda) \times[-1 / \lambda, 0]$.

- $x \in[2 /(3 \lambda), \lambda / 2)$ : Here $\hat{S}(x, y)=(1 / x-\lambda, 1 / y-\lambda)$ for $(x, y) \in \Omega^{*}$. Thus $[2 /(3 \lambda), \lambda / 2) \times[-\infty, 0)$ is sent to $\left[\phi_{1}, \lambda / 2\right) \times[-\infty,-\lambda]$.

- $x \in[\lambda / 2, \alpha)$ : Here again $\hat{S}(x, y)=(1 / x-\lambda, 1 / y-\lambda)$ for $(x, y) \in \Omega^{*}$. Then $[2 /(3 \lambda), \alpha) \times[-1 / R, 0]$ is sent to $\left[\phi_{l+1}, \phi_{1}\right) \times[-\infty,-R-\lambda]=$ $\left[\phi_{l+1}, \phi_{1}\right) \times\left[-\infty,-1 / L_{2}\right]$.

- $x \in[\alpha, 2 / \lambda)$ : Again, $\hat{S}(x, y)=(1 / x-\lambda, 1 / y-\lambda)$ for $(x, y) \in \Omega^{*}$ and $[\alpha, 2 / \lambda) \times\left[-1 /\left(\lambda-L_{2 l}\right), 0\right]$ is sent to $\left[-\lambda / 2, \phi_{l+1}\right) \times\left[-\infty,-\lambda-1 / L_{1}\right]$.

Combining the above, we get the first part of the assertion of the proposition.

4.3. Proof of Theorem 5: planar system is natural extension. We first recall some terminology and notation from [22]. Let $B$ be a set, and $T$ : $B \rightarrow B$ be a map. The pair $(B, T)$ is called a fibred system if the following three conditions are satisfied:

(a) There is a finite or countable set $I$ (called the digit set).

(b) There is a map $k: B \rightarrow I$. Then the sets $B(i)=k^{-1}(i)$ form a partition of $B$.

(c) The restriction of $T$ to any $B(i)$ is an injective map.

See [22, Definition 1.1.1]. From our definition of the mediant-Rosen map (see Definition 2), we naturally have $B=\mathbb{J}_{k}=[-\lambda / 2,2 / \lambda], T=S$, $I=\{i, i i, i i i, i v\}$, and $B(i)=[-\lambda / 2,-2 /(3 \lambda)), B(i i)=[-2 /(3 \lambda), 0)$, $B(i i i)=[0,2 /(3 \lambda))$, and $B(i v)=[2 /(3 \lambda), 2 / \lambda)$ (see also Figures 3 and 4$)$.

The pair $\left(B^{\#}, T^{\#}\right)$ is called a dual fibred system (or backward algorithm) with respect to $(B, T)$ if the following condition holds: $\left(k_{1}, \ldots, k_{n}\right)$ is an admissible block of digits for $T$ if and only if $\left(k_{n}, \ldots, k_{1}\right)$ is admissible for $T^{\#}$ (see Definition 21.1.1 in [22]). Furthermore,

$$
\begin{aligned}
D(x):=\left\{y \in B^{\#}: y \in B^{\#}\left(k_{1}, \ldots, k_{N}\right)\right. \text { if and only if } \\
\left.\qquad T^{-N}(x) \cap B\left(k_{N}, \ldots, k_{1}\right) \neq \emptyset, \text { for all } N \geq 1\right\}
\end{aligned}
$$

(see Definition 21.1.7 from [22]). The local inverse of the map $T: B(k) \rightarrow B$ is denoted by $V(k)$. Schweiger obtained the following theorem (see [22, Theorem 21.2.1]).

Theorem 6 (Schweiger). Consider the dynamical system $(\bar{B}, \bar{T})$ with $\bar{B}=\{(x, y): x \in B, y \in D(x)\}$, where $\bar{T}: \bar{B} \rightarrow \bar{B}$ is defined by

$$
\bar{T}(x, y)=\left(T(x), V^{\#}(k(x))(y)\right) .
$$

If $\bar{T}$ is measurable, then $(\bar{B}, \bar{T})$ equipped with the obvious product $\sigma$-algebra is an invertible dynamical system. Furthermore, if $K$ is a non-negative mea- 
surable function such that

$$
K(T x, y)\left|T^{\prime}(x)\right|=K\left(x, T^{\#} y\right)\left|\left(T^{\#}\right)^{\prime} y\right|
$$

then $K$ is an invariant density for this system. The dynamical system $(\bar{B}, \bar{T})$ is the natural extension of $(B, T)$.

In our setting we have $\bar{B}=\Omega^{*}$ by construction. So to apply Schweiger's theorem, we need to find a backward algorithm $\left(B^{\#}, T^{\#}\right)$ such that

$$
V^{\#}(k(x))(y)=M_{1}^{-1}(y) .
$$

Thus, on each $B^{\#}(\kappa)$ the map $T^{\#}$ is given by the inverse of the matrix giving the mediant-Rosen map on the corresponding $B(\kappa)$. Comparing with Definition 2, this map must be

$$
T^{\#}(y)= \begin{cases}\frac{-1}{y+\lambda}, & y \in B^{\#}(i), \\ \frac{-y}{\lambda y+1}, & y \in B^{\#}(i i), \\ \frac{y}{\lambda y+1}, & y \in B^{\#}(i i i), \\ \frac{1}{y+\lambda}, & y \in B^{\#}(i v) .\end{cases}
$$

Using the proofs of Propositions 2 and 3, we find the partition of $B^{\#}=$ $(-\infty, 0]$ (recall that $R=1$ in the even index case):

$$
\begin{aligned}
B^{\#}(i) & =[-\lambda,-1 / R), & B^{\#}(i i i) & =[-1 / \lambda, 0), \\
B^{\#}(i i) & =[-1 / R,-1 / \lambda), & B^{\#}(i v) & =(-\infty,-\lambda)
\end{aligned}
$$

(again, see Figures 3 and 4 ). An easy calculation shows that $V^{\#}(k(y))$ satisfies (9).

We find that $\left(B^{\#}, T^{\#}\right)$ is the dual fibred system with respect to $\left(\mathbb{J}_{k}, S\right)$. We already saw that $\frac{d x d y}{|x-y|^{2}}$ is an invariant measure for $\hat{S}$; note that this is compatible with the requirements on $K$ in Schweiger's theorem. Thus it follows that $\left(\Omega^{*}, \overline{\mathcal{B}}, \nu, \hat{S}\right)$ is the natural extension of the dynamical system $\left(\mathbb{J}_{k}, \mathcal{B}, \mu_{k}, S\right)$.

REMARK. Note that one could use the above method to verify that the system given in [3] is indeed the natural extension of the Rosen map. With this, as suggested by the referee, one can use the relationship between $S$ and $T$ to show that $\hat{S}$ is indeed the map giving the natural extension of $S$.

4.4. Ergodicity. We denote by $\hat{\mu}$ the measure with density function $1 /(x-y)^{2}$ with respect to Lebesgue measure, and by $\mu$ its marginal distribution on the first coordinate. 
Theorem 7. The dynamical system $\left(\Omega^{*}, \hat{S}, \hat{\mu}\right)$ is ergodic, and its entropy $h(\hat{S}, \hat{\mu})$ is equal to $(k-2) \pi^{2} /(2 k)$.

Proof. An easy calculation shows that

$$
\hat{S}^{k_{m}}(x, y)=\left(\left(M_{1} \cdots M_{k_{m}}\right)^{-1}(x),\left(M_{1} \cdots M_{k_{m}}\right)^{-1}(y)\right)
$$

and

$$
\left(M_{1} \cdots M_{k_{m}}\right)^{-1}(x)=T^{m}(x) .
$$

Thus, $\hat{S}^{k_{m}}$ is the induced transformation $\hat{S}_{\Omega_{0}}$ of $\hat{S}$ to $\Omega_{0}$, and is conjugate to $\mathcal{T}$ by the isomorphism $(x, y) \mapsto(x,-1 / y)$. Since $\mathcal{T}$ is ergodic (again, see [3]), so is $\hat{S}_{\Omega_{0}}$. In turn, this implies the ergodicity of $\hat{S}$.

The entropy $h(\hat{S}, \hat{\mu})$ of $(\hat{S}, \hat{\mu})$ is given by the entropy of its induced transformation on the region $\Omega_{0}$, as

$$
h(\hat{S}, \hat{\mu})=h\left(\hat{S}_{\Omega_{0}}, \hat{\mu}_{\Omega_{0}}\right) \hat{\mu}\left(\Omega_{0}\right),
$$

where $\hat{\mu}_{\Omega_{0}}$ is the restricted normalized measure of $\hat{\mu}$ to $\Omega_{0}$ (see [10]). Since $\left(\hat{S}_{\Omega_{0}}, \hat{\mu}_{\Omega_{0}}\right)$ is a natural extension of the Rosen map, and its entropy is

$$
C \frac{(k-2) \pi^{2}}{2 k}
$$

where $C$ is the normalizing constant of the invariant measure, i.e.,

$$
C^{-1}=\iint_{\Omega_{0}} \frac{d x d y}{|x-y|^{2}}
$$

(see [16]), the result follows.

The following result is an immediate consequence of Theorem 7 .

COROLlary 1 . The dynamical system $(S, \mu)$ is ergodic, and its entropy $h(S, \mu)$ is equal to $(k-2) \pi^{2} /(2 k)$.

REMARK. The density function with respect to Lebesgue measure $f$ of the measure $\mu$ is given by

which diverges at $x=0$.

$$
f(x)=\int_{\left\{y:(x, y) \in \Omega^{*}\right\}} \frac{d y}{(x-y)^{2}},
$$

Corollary 2. For a.e. $x \in \mathbb{I}_{k},\left\{v_{m, l}\left|x-u_{m, l} / v_{m, l}\right|: 1 \leq l \leq r_{m}-1\right.$, $m \geq 1\}$ is unbounded.

Proof. From the ergodicity of $\hat{S}$, for a.e. $(x, y) \in \Omega^{*}$, its forward $\hat{S}$-orbit is dense in $\Omega^{*}$. It follows that the distance between the second coordinates of $\hat{S}^{n}(x, y)$ and $\hat{S}^{n}(x,-\infty)$ tends to 0 . Thus the forward $\hat{S}$-orbit of $(x,-\infty)$ is also dense in $\Omega^{*}$. By Fubini's theorem, this holds for a.e. $x \in \mathbb{I}_{k}$. Because

$$
\hat{S}^{n}(x,-\infty)=\left(S^{n}(x),-q_{m} / v_{m, s}\right)
$$

for $n=k_{m}+s$ and by (4), we have the assertion. 
5. Proof of Theorem 4-exhibiting a witness. In this section, we use continued fraction methods to prove Theorem 1 . That is, we display the witness mentioned in the introduction. For each $k>3$, we call our witness $\tau_{0}$; this value is suggested by the geometry of our planar natural extension. Let

$$
\Theta\left(x, \frac{a}{c}\right)=c^{2}\left|x-\frac{a}{c}\right|
$$

for $\left(\begin{array}{l}a \\ c\end{array}\right) \in G_{k}$. Hereafter, when we write $a / c$ we assume that there exists a matrix $\left(\begin{array}{l}a \\ c\end{array} \cdot\right) \in G_{k}$. In case $a / c$ is equal to the $n$th mediant convergent of $x$ (with indexing as for (5)), we write $\Theta_{n}(x)$ instead of $\Theta(x, a / c)$.

The approach in both the even and odd index cases is quite similar, the odd case being (as usual with the $G_{k}$ ) a little more complicated. We give full detail of the even case, and outline the odd case.

5.1. Even index case: $k=2 l$. One of the main ingredients of the aforementioned Borel-type result from [9] is the fact that if we set

$$
\tau_{0}:=\left[\overline{(-1: 1)^{l-2},(-1: 2)}\right]=1-\lambda=-L_{l-1}, \quad \eta_{0}:=L_{1}=\frac{1}{\lambda+1}
$$

(here the bar indicates periodicity), the sequence

$$
\left(\tau_{i}, \eta_{i}\right):=\mathcal{T}^{i}\left(\tau_{0}, \eta_{0}\right) \quad \text { for } i \geq 0
$$

is purely periodic, with period length $l-1$. Here $\mathcal{T}: \Omega \rightarrow \Omega$ is the natural extension map from [3] (see (7)). Note that $\tau_{l-2}=-1 /(\lambda+1)=-L_{1}$, $\eta_{l-2}=\lambda-1=L_{l-1}$, and $\mathcal{T}\left(\tau_{l-2}, \eta_{l-2}\right)=\left(\tau_{0}, \eta_{0}\right)$. Furthermore, in [3] it was shown that if

$$
\left(t_{n}, v_{n}\right)=\mathcal{T}^{n}(x, 0) \quad \text { for } x \in[-\lambda / 2, \lambda / 2) \text { and } n \geq 0,
$$

then

$$
\theta_{n-1}(x)=\frac{v_{n}}{1+t_{n} v_{n}}, \quad \theta_{n}(x)=\frac{\left|t_{n}\right|}{1+t_{n} v_{n}}, \quad n \geq 1 .
$$

Due to this

$$
\theta\left(\tau_{i}, \eta_{i}\right):=\frac{\eta_{i}}{1+\tau_{i} \eta_{i}} \geq C(k)=\frac{1}{2}
$$

for $i \geq 0$ (with equality if $i \equiv 0(\bmod l-1)$ or $i \equiv l-2(\bmod l-1))$, and since $\theta_{i-1+n(l-1)}\left(\tau_{0}\right) \uparrow \theta\left(\tau_{i}, \eta_{i}\right)$ as $n \rightarrow \infty$, it follows that for any $C<C(k)=1 / 2$,

$$
\theta_{n}\left(\tau_{0}\right)<C \quad \text { for at most finitely many } n \geq 0 \text {. }
$$

Recall that $\Omega_{0}$ is an isomorphic copy of $\Omega$; for $i=0,1, \ldots, l-2$, the points $\left(\tau_{i}, \eta_{i}\right) \in \Omega$ correspond to the points $\left(\tau_{i}, K_{i+1}\right) \in \Omega_{0}$. Since the isomorphic copy of the system $\left(\Omega_{0}, \mathcal{T}\right)$ is induced from $\left(\Omega^{*}, \hat{S}\right)$, the $\hat{S}$-orbit of $\left(\tau_{0}, K_{1}\right)$ has cardinality at least $l-1$. In fact, this $\hat{S}$-orbit is also purely periodic, but is of cardinality $l$ : since $\tau_{i}<-2 /(3 \lambda)$ for $i=0,1, \ldots, l-3$, and $\tau_{l-2}>-2 /(3 \lambda)$, 
we have

$$
\hat{S}\left(\tau_{l-2}, K_{l-1}\right)=\hat{S}\left(\frac{-1}{\lambda+1}, \frac{1}{\lambda-1}\right)=(1,-1),
$$

and

$$
\hat{S}(1,-1)=\left(\frac{1}{1}-\lambda, \frac{1}{-1}-\lambda\right)=(1-\lambda,-1-\lambda)=\left(\tau_{0}, K_{1}\right) .
$$

If we set

$$
\left(T_{n}, V_{n}\right)=\hat{S}^{n}\left(\tau_{0},-\infty\right), \quad n \geq 0,
$$

it follows from (6), and the fact that (an isomorphic copy of) $\mathcal{T}$ is an induced transformation of $\hat{S}$, that $\left(\theta_{n}\left(\tau_{0}\right)\right)_{n \geq 0}$ is a subsequence of $\left(\Theta_{n}\left(\tau_{0}\right)\right)_{n \geq 0}$. In fact, the only points in the latter sequence which are not in the former are among the numbers $\Theta(1, y)$ with $-1<y<0$. For these numbers we have $\Theta(1, y)=1 /(1-y)>\Theta(1,-1)=1 / 2$. Consequently, for any $C<C(k)$ $=1 / 2$,

$$
\Theta_{n}\left(\tau_{0}\right)<C \quad \text { for at most finitely many } n \geq 0 .
$$

5.2. Odd index case: $k=2 l+3$. Analogously to the even index case, we set $\tau_{0}$ equal to the "left-top height" of $\Omega$, and $\eta_{0}$ equal to the lowest "height" of $\Omega$, i.e.,

$$
\tau_{0}:=-L_{2 l+1}=R-\lambda, \quad \eta_{0}:=L_{1}=\frac{1}{\lambda+1 / R},
$$

and we set

$$
\left(\tau_{i}, \eta_{i}\right):=\mathcal{T}^{i}\left(\tau_{0}, \eta_{0}\right) \quad \text { for } i \geq 0 .
$$

Again the sequence $\left(\tau_{i}, \eta_{i}\right)_{i \geq 0}$ is purely periodic, with period length $2 l$ (see [9]). In contrast to the even case, this sequence is more "complicated," with a kind of "double loop." On $\Omega_{0}$ the sequence corresponding to $\left(\tau_{i}, \eta_{i}\right)_{i \geq 0}$ is the (purely periodic) sequence $\left(\tau_{i}, K_{i+1}\right)_{i \geq 0}$ (see Figure 5 ).
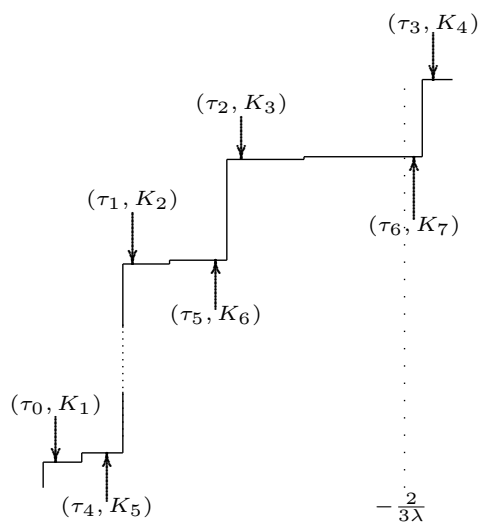

$$
-\frac{2}{3 \lambda}
$$

Fig. 5. The sequence $\left(\tau_{i}, K_{i+1}\right)_{i \geq 0}$ in $\Omega_{0}$ for $k=9$ 
As in the even case, $\bar{S}$ "picks up" a few extra points in the orbit of $\left(\tau_{0}, K_{1}\right)$, since both $\tau_{l}=-L_{1}$ and $\tau_{2 l}=-L_{2}$ are larger than $-2 /(3 \lambda)$. Similar to the even case, we find that for any $C<C(k)=1 /\left(2 \sqrt{\left(1-\lambda_{k} / 2\right)^{2}+1}\right)$,

$$
\Theta_{n}\left(\tau_{0}\right)<C \quad \text { for at most finitely many } n \geq 0 \text {. }
$$

6. Equality of Legendre and Lenstra constants. We define each of the Legendre and Lenstra constants for the mediant-Rosen maps and show their equality.

6.1. Definitions. Fix an index $k$, and suppose that there exists $\ell_{k}>0$ such that (i) for any $G_{k}$-irrational $x$ and any finite $G_{k}$-rational $a / c$,

$$
\left|x-\frac{a}{c}\right|<\frac{\ell_{k}}{c^{2}}
$$

implies $a / c$ is either a Rosen convergent $p_{n} / q_{n}$ for some $n \geq 0$, or a Rosen mediant convergent $u_{n, l} / v_{n, l}$ of $x$; and, (ii) for any $C>\ell_{k}$, there exist $x$ and $a / c$ such that

$$
\left|x-\frac{a}{c}\right|<\frac{C}{c^{2}}
$$

and $a / c$ is neither a Rosen convergent nor a mediant-Rosen convergent. Then we call $\ell_{k}>0$ the Legendre constant for mediant-Rosen convergents (of index $k$ ). The Legendre constant certainly exists for any index $k \geq 4$ because the Legendre constant for the Rosen continued fractions exists (see [21]), and the mediant-Legendre constant is certainly larger than or equal to it.

We again fix an index $k$, and now suppose that there exists $\mathcal{L}_{k}>0$ such that both: for any $0<t_{1}, t_{2}<\mathcal{L}_{k}$,

$$
\lim _{N \rightarrow \infty} \frac{\sharp\left\{1 \leq n \leq N: \Theta\left(M_{1} \cdots M_{n}(\infty), x\right)<t_{1}\right\}}{\sharp\left\{1 \leq n \leq N: \Theta\left(M_{1} \cdots M_{n}(\infty), x\right)<t_{2}\right\}}=\frac{t_{1}}{t_{2}}
$$

for a.e. $x \in \mathbb{J}$; and, for any $0<t_{2}<\mathcal{L}_{k}<t_{1}$,

$$
\lim _{N \rightarrow \infty} \frac{\sharp\left\{1 \leq n \leq N: \Theta\left(M_{1} \cdots M_{n}(\infty), x\right)<t_{1}\right\}}{\sharp\left\{1 \leq n \leq N: \Theta\left(M_{1} \cdots M_{n}(\infty), x\right)<t_{2}\right\}}<\frac{t_{1}}{t_{2}}
$$

for a.e. $x \in \mathbb{J}$. We call $\mathcal{L}_{k}$ the Lenstra constant for the mediant-Rosen convergents (of index $k$ ).

6.2. Legendre constant bounded above by Lenstra constant. The following is a direct consequence of the corollary of Section 2 in [16].

TheOREM 8. Fix any $t_{0}>0$. Then for any $t>0$ we have

$$
\lim _{Q \rightarrow \infty} \frac{\#\left\{a / c \in G_{k}(\infty): \Theta(a / c, x)<t, 0<c \leq Q\right\}}{\#\left\{a / c \in G_{k}(\infty): \Theta(a / c, x)<t_{0}, 0<c \leq Q\right\}}=\frac{t}{t_{0}}
$$

for a.e. $x$. Here we recall that $G_{k}(\infty)$ is the set of parabolic points of the Hecke group $G_{k}$. 
From this, we have the following result.

Proposition 4. The Legendre constant is less than or equal to the Lenstra constant, i.e., $\ell_{k} \leq \mathcal{L}_{k}$.

Proof. Let

$$
C(n, x, t)=\#\left\{j: 1 \leq j \leq n, \Theta\left(M_{1} \cdots M_{j}(\infty), x\right)<t\right\} .
$$

If $t$ is smaller than $\ell_{k}$, then we have

$$
\lim _{N \rightarrow \infty} \frac{\#\left\{a / c \in G_{k}(\infty): \Theta(a / c, x)<t, 0<c \leq q_{N}\right\}}{\#\left\{a / c \in G_{k}(\infty): \Theta(a / c, x)<t_{0}, 0<c \leq q_{N}\right\}}=\lim _{n \rightarrow \infty} \frac{C(n, x, t)}{C\left(n, x, t_{0}\right)}
$$

for almost every $x \in I$. But this implies that for each such $t$ and for each of these $x$, the limit as $N$ tends to infinity of the average of the counting function, $C(n, x, t) / N$, is a linear function in $t$. That is, $\mathcal{L}_{k} \geq \ell_{k}$.

6.3. Harder inequality. The idea of the following proof is to begin with an $x_{0}$ which is fairly well approximated by some $G_{k}$-rational not arising as a mediant map convergent. We then identify, in terms of the Rosen fraction expansion of this $x_{0}$, a whole cylinder set of $x$ all of which are fairly well approximated by such $G_{k}$-rationals. There is then a deficit in the numerator of the fraction of the fundamental equation (13). This then gives the desired inequality. We fix $t_{0}>0$ sufficiently small so that it is smaller than the Legendre constant associated to Rosen continued fractions.

Proposition 5. Let $C(n, x, t)$ be defined as in (14). For $t>\ell_{k}$ and a.e. $x \in \mathbb{I}_{k}$,

$$
\lim _{n \rightarrow \infty} \frac{C(n, x, t)}{C\left(n, x, t_{0}\right)}<\frac{t}{t_{0}}
$$

In particular, $\mathcal{L}_{k} \leq \ell_{k}$.

Proof. Fix $t$ such that $t>\ell_{k}$. Then there exist $x_{0} \in I$ and $p / q \in G_{k}(\infty)$ such that

$$
\Theta\left(x_{0}, p / q\right)<t \quad \text { with } p / q \text { unequal to any mediant convergent. }
$$

Consider the Rosen fraction expansions

$$
x_{0}=\left[\varepsilon_{1}: c_{1}, \ldots, \varepsilon_{n}: c_{n}, \ldots\right],
$$

and

$$
\frac{p}{q}=\left[\varepsilon_{1}^{\prime}: d_{1}, \ldots, \varepsilon_{l}^{\prime}: d_{l}\right]
$$

Since $p / q$ is not a mediant convergent, at least one of the following does not hold:

$$
\varepsilon_{j}=\varepsilon_{j}^{\prime} \text { for } 1 \leq j \leq l ; \quad c_{j}=d_{j} \text { for } 1 \leq j \leq l-1 ; \quad 1 \leq d_{l} \leq c_{l}-1 .
$$


We can choose a large integer $L$ such that

$$
\left.\left|y-\frac{p}{q}\right|<\frac{t}{q^{2}} \quad \text { (equivalently, } \Theta(y, p / q)<t\right)
$$

whenever

$$
y=\left[\varepsilon_{1}: c_{1}, \ldots, \varepsilon_{L}: c_{L}, \varepsilon_{L+1}^{\prime \prime}: c_{L+1}^{\prime \prime}, \varepsilon_{L+2}^{\prime \prime}: c_{L+2}^{\prime \prime}, \ldots\right] .
$$

We consider all such $y$. Now suppose that

$$
z=\frac{\eta_{1} \mid}{\mid a_{1}}+\frac{\eta_{2} \mid}{\mid a_{2}}+\cdots+\frac{\eta_{N} \mid}{\mid a_{N}+y}
$$

and

$$
\frac{P}{Q}=\frac{\eta_{1} \mid}{\mid a_{1}}+\frac{\eta_{2} \mid}{\mid a_{2}}+\cdots+\frac{\eta_{N} \mid}{\mid a_{N}}+\frac{p \mid}{\mid q} .
$$

For any such pair $(z, P / Q)$ we have

$$
\left|z-\frac{P}{Q}\right|<\frac{t}{Q^{2}}
$$

if $a_{N}$ is large enough, where the choice of $a_{N}$ depends only on $x_{0}, p / q$, and $L$. One checks that $P / Q$ is not a mediant convergent of $z$ (and thus in particular not a Rosen convergent). Fix some such $a_{N}$ and denote it by $a$, and $\eta_{N}$ by $\eta$.

Let $\mathcal{C}$ be the cylinder set of all $x$ such that the initial segment of the Rosen expansion of $x$ matches these:

$$
\mathcal{C}:=\left\{x \in I: x=\left[\eta: a, \varepsilon_{1}: c_{1}, \ldots, \varepsilon_{L}: c_{L}, \ldots\right]\right\} .
$$

By the above discussion, whenever $T^{n}(x) \in \mathcal{C}$ there exists a $G_{k}$-rational $P / Q$ such that

$$
\left|x-\frac{P}{Q}\right|<\frac{t}{Q^{2}} \quad \text { and } \quad q_{n-c} \leq Q<q_{n+c},
$$

where $c$ is a constant independent of $n$. Since the Rosen map is ergodic with respect to the invariant probability measure given in [3], the ergodic theorem applies and shows that for a.e. $x \in \mathbb{I}_{k}$, we have

$$
\lim _{N \rightarrow \infty} \frac{\#\left\{n \leq N: T^{n}(x) \in \mathcal{C}\right\}}{N}=\delta,
$$

where $\delta$ is the measure of $\mathcal{C}$ with respect to the invariant ergodic measure. In particular, this limit is positive.

Now let $\Xi_{N}(x)$ be the number of $P / Q \in G_{k}(\infty)$ such that

$$
\left|x-\frac{P}{Q}\right|<\frac{t}{Q^{2}}, \quad Q<q_{N}
$$


and $P / Q$ is neither a convergent nor a mediant convergent of $x$. From the above, we conclude that

$$
\liminf _{N \rightarrow \infty} \frac{\Xi_{N}}{C_{0}(N, x, t)}>0
$$

for a.e. $x \in \mathbb{I}_{k}$, where $C_{0}(N, x, t)=\#\left\{1 \leq n \leq N: \Theta\left(M_{1} \cdots M_{k_{n}}(\infty), x\right)<t\right\}$.

Now,

$$
\begin{aligned}
\limsup _{n \rightarrow \infty} \frac{C(n, x, t)}{C\left(n, x, t_{0}\right)} \\
\leq \limsup _{N \rightarrow \infty} \frac{\#\left\{a / c \in G_{k}(\infty): \Theta(x, a / c)<t, 0<c \leq q_{n}\right\}-\Xi_{N}(x)}{\#\left\{a / c \in G_{k}(\infty): \Theta(x, a / c)<t_{0}, 0<c \leq q_{N}\right\}} \\
\quad=\frac{t}{t_{0}}-\liminf _{N \rightarrow \infty} \frac{\Xi_{N}(x)}{\#\left\{a / c \in G_{k}(\infty): \Theta(x, a / c)<t_{0}, 0<c \leq q_{N}\right\}} \\
\leq \frac{t}{t_{0}}-\liminf _{N \rightarrow \infty} \frac{C_{0}(N, x, t)}{C_{0}\left(N, x, t_{0}\right)} \leq \frac{t}{t_{0}}-\delta<\frac{t}{t_{0}} .
\end{aligned}
$$

Consequently, the Lenstra constant of the mediant map cannot be larger than its Legendre constant.

We have thus demonstrated the equality of the Legendre and Lenstra constants for the mediant convergents.

7. Evaluating the Lenstra constant. In this section, we determine the exact value of the Lenstra constant, and hence of the Legendre also, for the mediant and the principal Rosen convergents. Note that for the principal Rosen convergents, the value of the Lenstra constant was stated-without proof - in Corollary 4.1 of [3].

7.1. Reduction to geometry of natural extension. First we consider the natural extension $\hat{T}$ of the Rosen continued fraction map $T$, defined as follows: the region of the natural extension $\Omega_{0}$ is given by (8), and for $(x, y) \in \Omega_{0}$, we define

$$
\hat{T}(x, y)=\left(\left(\begin{array}{cc}
-r(x) \lambda & \operatorname{sgn}(x) \\
1 & 0
\end{array}\right)(x),\left(\begin{array}{cc}
-r(x) \lambda & \operatorname{sgn}(x) \\
1 & 0
\end{array}\right)(y)\right) .
$$

This is bijective on $\Omega_{0}$ a.e., and the absolutely continuous invariant probability measure is given by

$$
C \frac{d x d y}{|x-y|^{2}}
$$

where $C$ is the normalizing constant (see [3] for the exact value of $C$ in both even and odd cases).

For $(x, y) \in \Omega_{0}$, we set $\left(x_{n}, y_{n}\right)=\hat{T}^{n}(x, y)$. In all that follows, we can extend to $y=-\infty$. In this case, (6) implies that $\theta_{n-1}(x)=1 /\left(x_{n}-y_{n}\right)$. (The 
similarity of this last denominator with the denominator in the expression for our invariant measure facilitates the following ergodic-theoretic approach.)

As observed in Section 4.4, the measure is ergodic. By the individual ergodic theorem and the standard approximation method (see say Chapter 4 in [7]), we have

$$
\lim _{N \rightarrow \infty} \frac{1}{N} \sharp\left\{n: 1 \leq n \leq N, x_{n}-y_{n}>t\right\}=C \iiint_{\left\{(x, y) \in \Omega_{0}: x-y>t\right\}} \frac{d x d y}{|x-y|^{2}}
$$

for any $t>0$ (a.e. $\left.(x, y) \in \Omega_{0}\right)$. Elementary calculus applies to show that the right hand side is equal to $C \lambda / t$ if $t$ is sufficiently large. By a simple calculation, we see that $\left|y_{n}-y_{n}^{\prime}\right| \rightarrow 0$ as $n \rightarrow \infty$ whenever $(x, y),\left(x, y^{\prime}\right) \in \Omega_{0}$. This implies that if (15) holds for $(x, y)$, then it also holds for $\left(x, y^{\prime}\right)$. Thus we get (15) for a.e. $x \in \mathbb{I}_{k}$ and any $y$ such that $(x, y) \in \Omega_{0}$; hence the property also holds for those values of $x$ and with $y=-\infty$. Therefore,

$$
\lim _{N \rightarrow \infty} \frac{1}{N} \sharp\left\{n: 1 \leq n \leq N, \theta_{n}(x)<c\right\}=C c
$$

if $c$ is sufficiently small (a.e. $x$ ). Thus the Lenstra constant for the Rosen fractions is the infimum of those $t>0$ for which

$$
\iint_{\left\{(x, y) \in \Omega_{0}: x-y>t\right\}} \frac{d x d y}{|x-y|^{2}}=\frac{\lambda}{t} .
$$

It is easily seen (cf. Figures 3 and 4 ) that this equals the infimum $t_{0}$ of those $t>0$ for which the points on the line segment

$$
y=x+t, \quad x \in \mathbb{I}_{k},
$$

are all in $\Omega_{0}$.

Now for the mediant-Rosen convergents, analogous arguments apply. We use the ratio ergodic theorem (see say Ch. 3 of [1]), instead of the individual ergodic theorem, and we obtain the completely analogous conclusion, i.e., $\mathcal{L}_{k}$ is the infimum $t_{1}$ of $t$ such that

$$
\iint_{\left\{(x, y) \in \Omega^{*}: x-y>t\right\}} \frac{d x d y}{|x-y|^{2}}=\frac{\lambda}{t} .
$$

We consider the even and the odd indices cases separately.

7.2. Even index case: $k=2 l$. First we show that the Lenstra constant for the Rosen convergents is $\lambda /(\lambda+2)$, confirming Corollary 4.1 of [3].

To find $t_{0}$ with property (18), it is enough to check the lines of slope 1 passing through the interior corners of $\Omega_{0}$. The associated equations are

$$
\left\{\begin{array}{l}
y=x-\left(1 / L_{j}+\phi_{j}\right), \quad 1 \leq j \leq l-1 \\
y=x-(1-\lambda / 2)
\end{array}\right.
$$


From these, we see that $t_{0}=\max \left\{1 / L_{j}+\phi_{j}(1 \leq j \leq l-1), 1-\lambda / 2\right\}$. Since it follows that

$$
L_{j}=\frac{1}{\lambda-L_{j-1}} \quad \text { and } \quad \phi_{j}=-\frac{1}{\phi_{j-1}}-\lambda \text {, }
$$

$$
\frac{1}{L_{j}}+\phi_{j}=\frac{1 / L_{j-1}+\phi_{j-1}}{\left|\phi_{j-1} / L_{j-1}\right|}
$$

for $2 \leq j \leq l-1$. Because $\left(1 / L_{j}\right)_{j=0}^{l-1}$ and $\left(\left|\phi_{j}\right|\right)_{j=0}^{l-1}$ are decreasing sequences, the maximum is either $1 / L_{1}+\phi_{1}, 1 / L_{l-1}+\phi_{l-1}$, or $1-\lambda / 2$. Now recall that $\phi_{l-1}=0$ and $L_{l-1}=\lambda-1$ and $\lambda \geq \sqrt{2}$. These yield the estimate $(\lambda+2) / \lambda=1 / L_{1}+\phi_{1} \geq 1 / L_{l-1}+\phi_{l-1}$. Note that it is easy to show that $(\lambda+2) / \lambda=1 / L_{1}+\phi_{1}>1-\lambda / 2$. Consequently, $t_{0}=1 / L_{1}+\phi_{1}=(\lambda+2) / \lambda$, and the result holds.

The result for the mediant-Rosen convergents is the following.

Proposition 6. The Lenstra constant for the mediant-Rosen convergents is $\lambda-1$ when the index is even and not equal to 4 . If $k=4$, then the Lenstra constant is equal to $\sqrt{2} / 2$.

Proof. It is obvious that the measure $d x d y /|x-y|^{2}$ is invariant under the translation $(x, y) \mapsto(x+z, y+z)$ for any real number $z$. We translate the set $J_{l+1} \times \bar{K}_{l+1}$ by $-\lambda$. Then the image is $\left[\phi_{0}, \phi_{1}\right) \times\left[-1 / L_{1},-1 / L_{1}+1\right)=$ $\left[\phi_{0}, \phi_{1}\right) \times[-\lambda-1,-\lambda)$ and we see that $-\lambda<-1 / L_{2}=-\lambda+1 /(\lambda+R)$. This shows that for the mediant case, we can get $t_{1}$ by $\max \left\{-\lambda+\phi_{1}\right.$, $\left.1 / L_{j}+\phi_{j}(2 \leq j \leq l-1), \lambda / 2\right\}$. Similarly to the above, the maximum is given by either $\lambda+\phi_{1}, 1 / L_{2}+\phi_{2}, 1 / L_{l-1}+\phi_{l-1}=1 /(\lambda-1)$, or $\lambda / 2$. Thus we get $t_{1}=1 /(\lambda-1)$ when $l \geq 3$ (see Figure 6 ). If $l=2$, a simple calculation shows that $t_{1}=\sqrt{2}+1$.

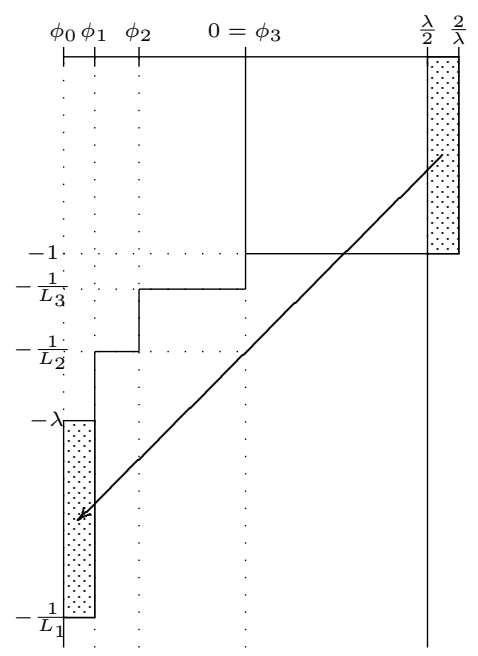

Fig. 6. Translation of $J_{l+1} \times \bar{K}_{l+1}$ by $-\lambda$. Here $k=8$. 
7.3. Odd index case: $k=2 l+3$. Here also we first confirm Corollary 4.1 of [3]: the Lenstra constant of the Rosen convergents equals $R /(R+1)$.

The idea of the calculation is the same as in the even case. Considering the slope 1 lines through the corners of $\Omega_{0}$, we find that

$$
t_{0}=\max \left\{\frac{1}{L_{2 j}}+\phi_{j}(1 \leq j \leq l), \frac{1}{L_{2 j-1}}+\phi_{l+j}(1 \leq j \leq l+1), \frac{1}{R}+\frac{\lambda}{2}\right\} .
$$

Since

$$
\phi_{j+1}=-\frac{1}{\phi_{j}}-\lambda \quad \text { for } 0 \leq j<l, l+1 \leq j<2 l+1
$$

and

$$
L_{j+2}=\frac{1}{\lambda_{j}-L_{j}} \quad \text { for } 1 \leq j \leq 2 l-1,
$$

we have

$$
\phi_{j+1}+\frac{1}{L_{2(j+1)}}=\frac{\phi_{j}+\frac{1}{L_{2 j}}}{-\phi_{j} \cdot \frac{1}{L_{2 j}}}
$$

and

$$
\phi_{l+j+1}+\frac{1}{L_{2 j+1}}=\frac{\phi_{l+j}+\frac{1}{L_{2 j-1}}}{-\phi_{l+j} \cdot \frac{1}{L_{2 j-1}}}
$$

for $1 \leq j \leq l-1$. Moreover, $\phi_{l+1}(=1-\lambda)=-1 / \phi_{l}-2 \lambda$ and $L_{1}=$ $1 /\left(2 \lambda-L_{l}\right)$ implies

$$
\phi_{l}+\frac{1}{L_{2 l}}=\frac{\phi_{l}+\frac{1}{L_{2 l}}}{-\phi_{l} \cdot \frac{1}{L_{2}}} .
$$

Again, $\left(\left|\phi_{j}\right|: 1 \leq j \leq l+1\right),\left(\left|\phi_{l+j}\right|: 1 \leq j \leq l+1\right)$, and $\left(1 / L_{j}\right.$ : $1 \leq j \leq 2 l-1)$ are decreasing sequences. So the above maximum is equal to

$$
\begin{aligned}
& \max \left\{\phi_{1}+\frac{1}{L_{2}}, \phi_{l+1}+\frac{1}{L_{1}},\right.\left.\phi_{2 l+1}+\frac{1}{L_{2 l+1}}, \frac{1}{R}+\frac{\lambda}{2}\right\} \\
&=\max \left\{\frac{2}{\lambda}+R, \frac{R+1}{R}, \frac{1}{\lambda-R}, \frac{1}{R}+\frac{\lambda}{2}\right\} .
\end{aligned}
$$

Due to the facts that $R^{2}+(2-\lambda) R-1=0$ and $\lambda / 2<R<1$, we see that the maximum is equal to $\frac{R+1}{R}$, and the result follows.

For the mediant-Rosen convergents, we have the following.

Proposition 7. In case of odd index $k$, the Lenstra constant for the mediant-Rosen convergents is $\lambda-R$.

Proof. We translate $\left(J_{2 l+3} \times \bar{K}_{2 l+3}\right) \cup\left(J_{2 l+4} \times \bar{K}_{2 l+4}\right)$ by $-\lambda$ (see Figure 7$)$. Then its image is

$$
\left[-\lambda / 2, \phi_{l+1}\right) \times[-1 / R-\lambda,-\lambda) \cup\left[\phi_{l+1}, 2 / \lambda-\lambda\right) \times[-R-\lambda,-\lambda) .
$$




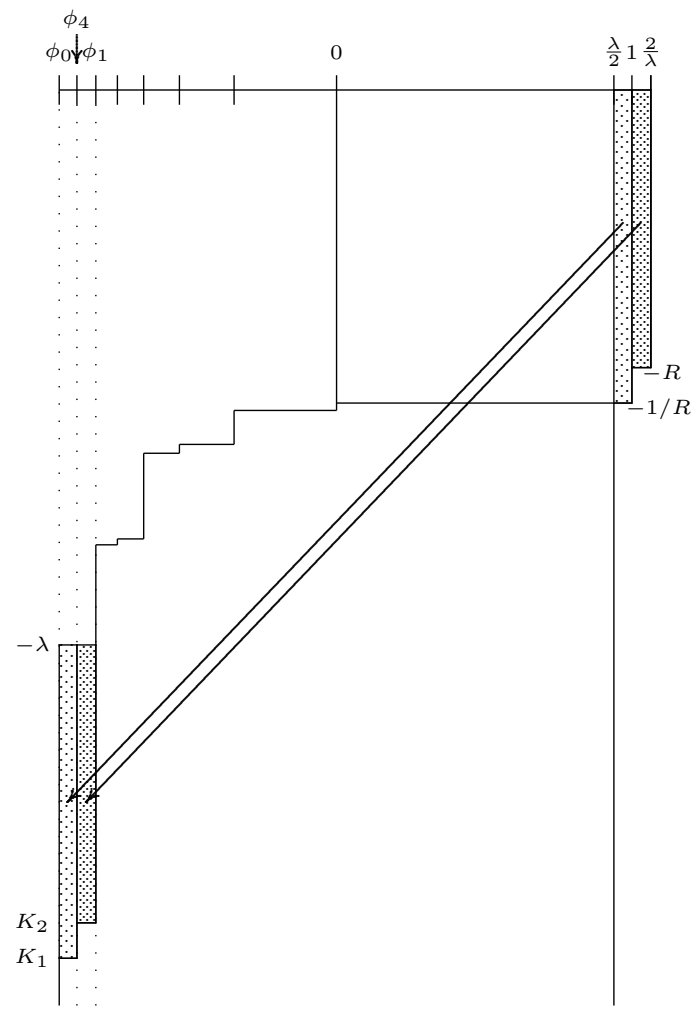

Fig. 7. Translation of $\left(J_{2 l+3} \times \bar{K}_{2 l+3}\right) \cup\left(J_{2 l+4} \times \bar{K}_{2 l+4}\right)$ by $-\lambda$. Here $k=9$.

This fits exactly on top of

$$
\begin{aligned}
J_{1} \times \hat{K}_{1} \cup J_{2} \times \hat{K}_{2}= & {\left[-\lambda / 2, \phi_{l+1}\right) \times[-\infty,-1 / R-\lambda) } \\
& \cup\left[\phi_{l+1}, 2 / \lambda-\lambda\right) \times[-\infty,-R-\lambda)
\end{aligned}
$$

(note that $\phi_{l+1}=1-\lambda$ ). These translations cover up the interior vertices contributing $1 / L_{2}+\phi_{1}$ and $1 / L_{1}+\phi_{l+1}$ to the set of $(20)$ and yield a new vertex giving $\phi_{1}+\lambda=2 / \lambda$. Thus we have

$t_{1}=\max \left\{\frac{2}{\lambda}, \frac{1}{L_{2 j}}+\phi_{j}(2 \leq j \leq l), \frac{1}{L_{2 j-1}}+\phi_{l+j}(2 \leq j \leq l+1), \frac{1}{R}+\frac{\lambda}{2}\right\}$.

This is the same as

$$
\max \left\{\frac{2}{\lambda}, \frac{1}{L_{4}}+\phi_{2}, \frac{1}{L_{2 l}}+\phi_{l}, \frac{1}{L_{3}}+\phi_{l+2}, \frac{1}{L_{2 l+1}}+\phi_{2 l+1}, \frac{\lambda}{2}\right\} .
$$

One has the following relations: 


$$
\begin{aligned}
\frac{1}{L_{4}}+\phi_{2} & =\frac{1}{\lambda-2 / \lambda}-\frac{1}{\lambda+R}, \\
\frac{1}{L_{2 l}}+\phi_{l} & =\frac{R}{\lambda R-1}-\frac{1}{\lambda+1}, \\
\frac{1}{L_{3}}+\phi_{l+2} & =\frac{1}{\lambda-1}-\frac{R}{\lambda R+1}, \\
\frac{1}{L_{2 l+1}}+\phi_{2 l+1} & =\frac{1}{\lambda-R} .
\end{aligned}
$$

Here $1 / L_{4}+\phi_{2}$ and $1 / L_{2 l}+\phi_{l}$ do not appear in the above when $k=5$. After some calculation, we see the maximum is $1 /(\lambda-R)$. In order to see that this is indeed the case, note that we obviously have $\lambda / 2<2 / \lambda$; moreover,

$$
\frac{2}{\lambda}<\frac{1}{\lambda-R}
$$

follows from $\lambda / 2<R$. Since $R^{2}+(2-\lambda) R-1=0$ and $\lambda \geq(1+\sqrt{5}) / 2$, we have

$$
\frac{1}{\lambda-1}-\frac{R}{\lambda R+1} \leq \frac{1}{\lambda-R}
$$

We see that

$$
\frac{1}{\lambda-2 / \lambda}-\frac{1}{\lambda+R}<\frac{1}{\lambda-R}
$$

when $k>5$. Here we have used the fact that $\lambda^{2}>4-R^{2}$ for $k>5$, which has to be checked somehow. Because $\lambda$ and $R$ are increasing as $k$ increases, it is sufficient to prove it for $k=7$.

Finally, we show that

$$
\frac{R}{\lambda R-1}-\frac{1}{\lambda+1}<\frac{1}{\lambda-2 / \lambda}-\frac{1}{\lambda+R}
$$

for $k \geq 7$. This inequality is equivalent to

$$
\frac{1}{\lambda-1 / R}+\frac{1}{\lambda+R}<\frac{\lambda}{\lambda^{2}-2}+\frac{1}{\lambda+1} .
$$

Since $R-1 / R=\lambda-2$, this is equivalent to

$$
\frac{3 \lambda-1}{\lambda^{2}+(\lambda-2) \lambda-1}<\frac{2 \lambda^{2}+\lambda-2}{\lambda^{3}+\lambda^{2}-2 \lambda-2} .
$$

Note that both denominators are positive (for $\lambda>\sqrt{3}$ ). The last inequality follows from

$$
\lambda^{4}-3 \lambda^{3}+5 \lambda-2>0 \quad \text { for } \lambda>\sqrt{3}
$$

Acknowledgments. We thank the referee for a careful reading and sound advice for improving the presentation of these results: in particular for insisting on clarifying material which led us to include Subsection 4.3. 
The second author was supported by Bezoekersbeurs B 61-620 of the Nederlandse Organisatie voor Wetenschappelijk Onderzoek (NWO). The first and third author thank the Center of Excellence of Keio University.

\section{References}

[1] J. Aaronson, An Introduction to Infinite Ergodic Theory, Amer. Math. Soc., 1997.

[2] W. Bosma, H. Jager, and F. Wiedijk, Some metrical observations on the approximation by continued fractions, Indag. Math. 45 (1983), 353-379.

[3] R. Burton, C. Kraaikamp, and T. A. Schmidt, Natural extensions for the Rosen fractions, Trans. Amer. Math. Soc. 352 (2000), 1277-1298.

[4] K. Dajani and C. Kraaikamp, Ergodic Theory of Numbers, Carus Math. Monogr. 29, Math. Assoc. Amer., Washington, DC, 2002.

[5] A. Haas, The distribution of geodesic excursions out the end of a hyperbolic orbifold and approximation with respect to a Fuchsian group, Geom. Dedicata 116 (2005), $129-155$.

[6] A. Haas and C. Series, The Hurwitz constant and Diophantine approximation on Hecke groups, J. London Math. Soc. 34 (1986), 219-234.

[7] M. Iosifescu and C. Kraaikamp, Metrical Theory of Continued Fractions, Math. Appl. 547, Kluwer, Dordrecht, 2002.

[8] S. Ito, Algorithms with mediant convergents and their metrical theory, Osaka J. Math. 26 (1989), 557-578.

[9] C. Kraaikamp, T. A. Schmidt, and I. Smeets, Tong's spectrum for Rosen continued fractions, J. Théor. Nombres Bordeaux 19 (2007), 641-661.

[10] U. Krengel, Entropy of conservative transformations, Z. Wahrsch. Verw. Gebiete 7 (1967), 161-181.

[11] J. Lehner, Discontinuous Groups and Automorphic Functions, Math. Surveys Monogr. 8, Amer. Math. Soc., 1964.

[12] -, Diophantine approximation on Hecke groups, Glasgow Math. J. 27 (1985), $117-127$.

[13] - , The local Hurwitz constant and diophantine approximation on Hecke groups, Math. Comp. 55 (1990), 765-781.

[14] H. Nakada, Metrical theory for a class of continued fraction transformations, Tokyo J. Math. 4 (1981), 399-426.

[15] - Continued fractions, geodesic flows and Ford circles, in: Algorithms, Fractals and Dynamics, Y. Takahashi (ed.), Plenum, 1995, 179-191.

[16] - On the Lenstra constant associated to the Rosen continued fractions, J. Eur. Math. Soc., to appear.

[17] H. Nakada, S. Ito, and S. Tanaka, On the invariant measure for the transformations associated with some real continued-fractions, Keio Engrg. Rep. 30 (1977), no. 13, 159-175.

[18] R. Natsui, On the interval maps associated to the $\alpha$-mediant convergents, Tokyo J. Math. 27 (2004), 87-106.

[19] V. A. Rokhlin, Exact endomorphisms of Lebesgue spaces, Izv. Akad. Nauk SSSR Ser. Mat. 25 (1961), 499-530 (in Russian); English transl.: Amer. Math. Soc. Transl. Ser. (2) 39 (1964), 1-36.

[20] D. Rosen, A class of continued fractions associated with certain properly discontinuous groups, Duke Math. J. 21 (1954), 549-563. 
[21] D. Rosen and T. A. Schmidt, Hecke groups and continued fractions, Bull. Austral. Math. Soc. 46 (1992), 459-474.

[22] F. Schweiger, Ergodic Theory of Fibred Systems and Metric Number Theory, Clarendon Press, Oxford, 1995.

Technische Universiteit Delft

and Thomas Stieltjes Institute of Mathematics

EWI, Mekelweg 4

2628 CD Delft, the Netherlands

E-mail: c.kraaikamp@tudelft.nl

Oregon State University

Corvallis, OR 97331, U.S.A.

E-mail: toms@math.orst.edu
Department of Mathematics

Keio University

Yokohama, Japan

E-mail: nakada@math.keio.ac.jp

Received on 24.8.2007

and in revised form on 23.1.2009 